\title{
Reconstructing the primordial power spectrum from the CMB
}

\author{
Christopher Gauthier and Martin Bucher \\ Laboratoire APC, Université Paris Diderot, Bâtiment Condorcet, \\ 10 rue Alice Domon et Léonie Duquet, 75205 Paris Cedex 13, France
}

(Dated: 11 September 2012)

\begin{abstract}
We propose a straightforward and model independent methodology for characterizing the sensitivity of CMB and other experiments to wiggles, irregularities, and features in the primordial power spectrum. Assuming that the primordial cosmological perturbations are adiabatic, we present a function space generalization of the usual Fisher matrix formalism, applied to a CMB experiment resembling Planck with and without ancillary data. This work is closely related to other work on recovering the inflationary potential and exploring specific models of non-minimal, or perhaps baroque, primordial power spectra. The approach adopted here, however, most directly expresses what the data is really telling us. We explore in detail the structure of the available information and quantify exactly what features can be reconstructed and at what statistical significance.
\end{abstract}




\section{INTRODUCTION}

In the last decade, CMB experiments have created new opportunities for studying the early universe. With the data obtained by WMAP [1] and the promise of even more accurate CMB measurements by Planck [2] in addition to the several current and future ground and balloon based experiments, the data will become even more constraining and it will be interesting to see whether the simple minimal model that seems to be able to explain all the present data survives or whether a new layer of complexity is discovered.

According to our current best understanding of primordial cosmology, the universe underwent a period of extremely rapid expansion known as inflation [3-6]. This model was initially proposed to solve several problems with the standard big bang theory. In addition, inflation was found to make quantitative predictions concerning the primordial cosmological perturbations [7-10]. In the simplest of these models, inflation predicts that the primordial fluctuations in primordial power spectrum (PPS) should be Gaussian with a nearly scale invariant spectrum. The most common method of interpreting the CMB data assumes a modest number of cosmological parameters (for example, in the WMAP 7-year analysis the parameters adopted are $\left.H_{0}, \omega_{b}, \omega_{c}, \Omega_{\Lambda}\right)$ and a parameterized form for the primordial power

spectrum, for example $P(k)=A\left(k / k_{0}\right)^{n_{s}-1}$ or perhaps $P(k)=A\left(k / k_{0}\right)^{\left(n_{s}-1-\beta \ln \left(k / k_{0}\right)\right)}$ where the parameter $\beta$ denotes the running of the spectral index. In this paper we adopt as a fiducial reference model the WMAP 7-year best fit [1], for which the parameters are $h=0.703$, $\omega_{b}=h^{2} \Omega_{b}=0.02227, \omega_{c}=h^{2} \Omega_{c}=0.1116, \tau=0.085, A=2.42 \times 10^{-9}$, and $n_{S}=0.966$. Here the somewhat arbitrary pivot scale has been set to $k_{0}=2.0 \times 10^{-3} \mathrm{Mpc}^{-1}$. Furthermore we assume that there are no tensor perturbations nor running in the scalar spectral index (i.e. $r=0$ and $\beta=0.0$ ) and also that the universe is exactly flat. It is encouraging to learn that a simple model of this sort can provide an adequate and plausible explanation of the presently available data, and following this general approach, one can construct more complicated models and compare these based on relative likelihood and ideas about model comparison.

However the drawback of an analysis comparing parameterized models to the data and among themselves is that which aspects of the model actually enter into the likelihood remains hidden inside a black box. If for example we show plots of constraints on $n_{s}$ or the running $\beta$, these plots do not show at what $k$ measurements have been made. The 
purpose of this paper is to enter into this black box and show numerically what exactly is being measured, in particular with respect to the primordial power spectrum. For simplicity we assume that the perturbations are adiabatic and Gaussian, although generalizing the treatment here to isocurvature perturbations is in principle straightforward.

Rather than developing a suite of fancy parametric models with lots of bells and whistles, we approach the problem more in the spirit of the method dubbed 'exploratory data analysis' by John Tukey (see for example [11]) where the aim is to devise methods to make manifest patterns in the data and what is being constrained and where, the purpose being to uncover the unexpected. This approach stands in contrast to the complementary approach where precise models with well-defined prior information are set forth as hypotheses and optimal statistical tests to discriminate between them are sought. In fact the use of priors in the paper is more formal and metaphorical, because for our purposes meaningful prior information is lacking. The priors serve more as regulators to suppress noise where there is seemingly no relevant information.

In the past a number of approaches have been proposed to search for features in the primordial power spectrum inferred from the CMB. One general class of approaches consists of parametric models, where $P(k)$ is modeled using a family of functions having a fixed and well-defined number of adjustable parameters. Bridle et al. [12], for example, analyzed the WMAP first-year results using a model with $\mathrm{N}$ knots equally spaced in $\ln (k)$ between a $k_{\min }$ and $k_{\max }$ where $\ln (P(k))$ is interpolated linearly between $N$ control points or knots. The number of knots admitted is a measure of the model complexity, and as is well-known, when comparing models it is necessary to compensate for the better fit due to fitting away noise when more degrees of freedom are present. This can be done using standard techniques (e.g., a simple $\chi^{2}$ analysis, the Aikake information criterion (AIC) [13], the Bayesian information criterion (BIC) $[14]^{1}$, etc). One disadvantage of this approach is that the placement of the bin boundaries, which is arbitrary, influences the conclusions. The sensitivity to a sharp feature having a width comparable to the bin width depends on where the feature is situated relative to the bin boundaries. Interesting variants have been proposed. Hamann et al. have proposed allowing the knot positions not to be fixed but rather parameters of the model

1 Care must be taken when applying the BIC to actual data because of the asymptotic nature of its derivation. It is often asked what value to use for $n_{\text {data }}$ in the $\log \left(n_{\text {data }}\right)$ term appearing in the BIC. Is it the number of $C_{\ell}$ or the total number of measurements? A careful analysis starting from a Bayesian prior reveals that while the BIC obtains the right scaling as $n_{\text {data }} \rightarrow \infty$, there is another prefactor that does not vary with $n_{\text {data }}$ that is ignored. Without knowing this prefactor, which would required a specific Bayesian prior, it is not possible to address this question. In reality, the factor $\operatorname{should}$ be $\ln \left(n_{\text {data }} / \bar{n}\right)$ where $\bar{n}$ depends on the overall relative likelihood of the models in the prior. 
[15]. Although this approach introduces nonlinearity into the model, it allows localized sharp features to be modeled by placing control points only where they are needed. In this way models giving a good fit to a single sharp feature are significantly less penalized than in the approach of Bridle et al. and others [12, 16].

Another class of approaches may be described as penalized likelihood methods. In this class of methods the function space for $P(k)$ includes many more parameters than desired. There is either a very large or infinite number of degrees of freedom. Model complexity is limited by penalizing very rough or rapidly varying functions. The log likelihood is modified to include a roughness penalty for example as in the following form:

$$
\ln L(\mathbf{d}, P(k), \boldsymbol{\beta})+\lambda \int d(\ln [k])\left(\frac{\partial^{2}(\ln P)}{\partial(\ln k)^{2}}\right)^{2} .
$$

Here we may appeal to Bayesian doctrine by describing the second term as a prior probability over the function space of $P(k)$. An example of this approach applied to the WMAP data can be found in [17]. However in practice this term rarely corresponds to any actual prior belief. Rather it is chosen a posteriori to obtain a meaningful result and depends on the quality of the data. The form of the roughness penalty in eqn. (1) is somewhat arbitrary and other reasonable forms have been proposed. We could also have used the square of the first rather than the second derivative, although this choice would have the drawback of slightly favoring an overall flatter spectrum. Also the weighting as a function of $\ln (k)$ and what is assumed for $k$ smaller and larger than where there is data involves arbitrary or subjective choices. The challenge of choosing the regularization parameter $\lambda$ involves finding the right compromise between underfitting and overfitting the data. There exists an abundant literature on how to make an optimal choice for $\lambda$ rapidly surveyed below. However in our view it does not suffice to find the optimal $\lambda$, because this information does not tell us which features of the reconstruction are to be believed and which are to be regarded as most likely explained as statistical fluctuations. The approach adopted in the paper is to study and characterize precisely the properties of the reconstruction for a variety of reasonable values for the smoothing parameter.

Tocchini-Valentini et al. [18, 19] adopted a penalized likelihood similar to that used in this paper, which was applied to the WMAP data finding some evidence for statistically significant features. In this paper we account for uncertainties in the cosmological parameters rather than fixing them and adopt a frequentist approach to the error analysis because we 
do not believe that the prior implicit in the smoothing penalty deserves to be interpreted as an actual prior belief or probability measure on the function space of possible power spectra.

Many reconstruction procedures can in some cases yield reconstructed primordial power spectra that take negative values in some places, which is clearly unphysical. This can occur when a quadratic approximation to the likelihood is made or when there is a superimposed noise component. Hamann et al. [15] (see also [20]) carried out a modified Lucy-Richardson deconvolution of the primordial power spectrum from CMB data. Lucy [21] and Richardson 22] introduced an algorithm widely used for image deconvolution having the property that the reconstruction is always positive. The original Lucy-Richardson algorithm was derived from a likelihood assuming a Poisson noise for the number counts. If there are some counts in a cell, an infinitely improbable likelihood results as the predicted average count tends to zero, quite unlike in a Gaussian, where a negative average count would not be excluded by the form of the likelihood. Unfortunately the CMB likelihood does not fit into this mold. Modifications to L-R algorithm have been proposed to take into account the properties of the CMB likelihood. However the modified L-R algorithm cannot be derived rigorously starting from a CMB likelihood. Hamann et al. overcome this difficulty by carrying out a frequentist analysis of the reconstructed power spectra, treating the reconstruction as a black box and characterizing its properties through Monte Carlo experiments.

$\mathrm{Hu} 223$ and Leach 24] explore an approach based on principal component analysis. A principal component analysis ranks directions of deviation with respect to a fiducial power spectrum using the eigenvalues and eigenvectors of the Fisher information matrix. The decomposition into eigenmodes, however, relies on a notion of length (or metric) for this space of linearized deviations, and thus entails some arbitrariness ${ }^{2}$ Peiris et al. [17, 25, 26] explore a penalized likelihood using 'cross-validation' to select an optimal degree of smoothing. A variety of other approaches have been proposed including wavelets (Mukherjee et al. [27-29]) and 'cosmic inversion' (Kogo et al. [30], Nagata et al. [31, 32] and Ichiki et al. [33]).

Vazquez et al. [34] present an interesting analysis based on Bayesian model comparison. A prior distribution is constructed for a linear interpolation of $\ln (P)$ as a function of $\ln (k)$ using a variable number of nodes whose positions are free, yielding a posterior distribution among the models. Using data from WMAP combined with ACT and SDSS LRG data, the

${ }^{2}$ A quadratic form is a linear mapping from a linear space into its dual and not into itself. Therefore a metric tensor is required to define a natural equivalence between the linear space and its dual. 
authors conclude that an $n_{S}=1$ Harrison-Zeldovich-Peebles spectrum is 'strongly' excluded within a Bayesian framework, using the terminology of Jeffreys.

This paper systematically studies and characterizes the trade-offs between bias and variance (i.e., noise) in the choice of smoothing scheme. Rather than espousing a particular philosophy leading to a unique solution, we show in a quantitative way the results for a range of choices. Which is best depends on what type of features one is trying to reconstruct.

The organization of the paper is as follows. Section 2 presents a detailed description of the constraints on the primordial power spectrum assuming a Planck-like experiment based on the Fisher information kernel. The information loss due to uncertainty in the determination of the other cosmological parameters is described. Section 3 describes the power spectrum construction first for the simplified case where the other cosmological parameters are fixed and then including their uncertainties. Section 4 characterizes the noise of the reconstruction and with what statistical significance various model features can be reconstructed. Finally Section 5 presents some concluding remarks. 


\section{FISHER INFORMATION KERNEL}

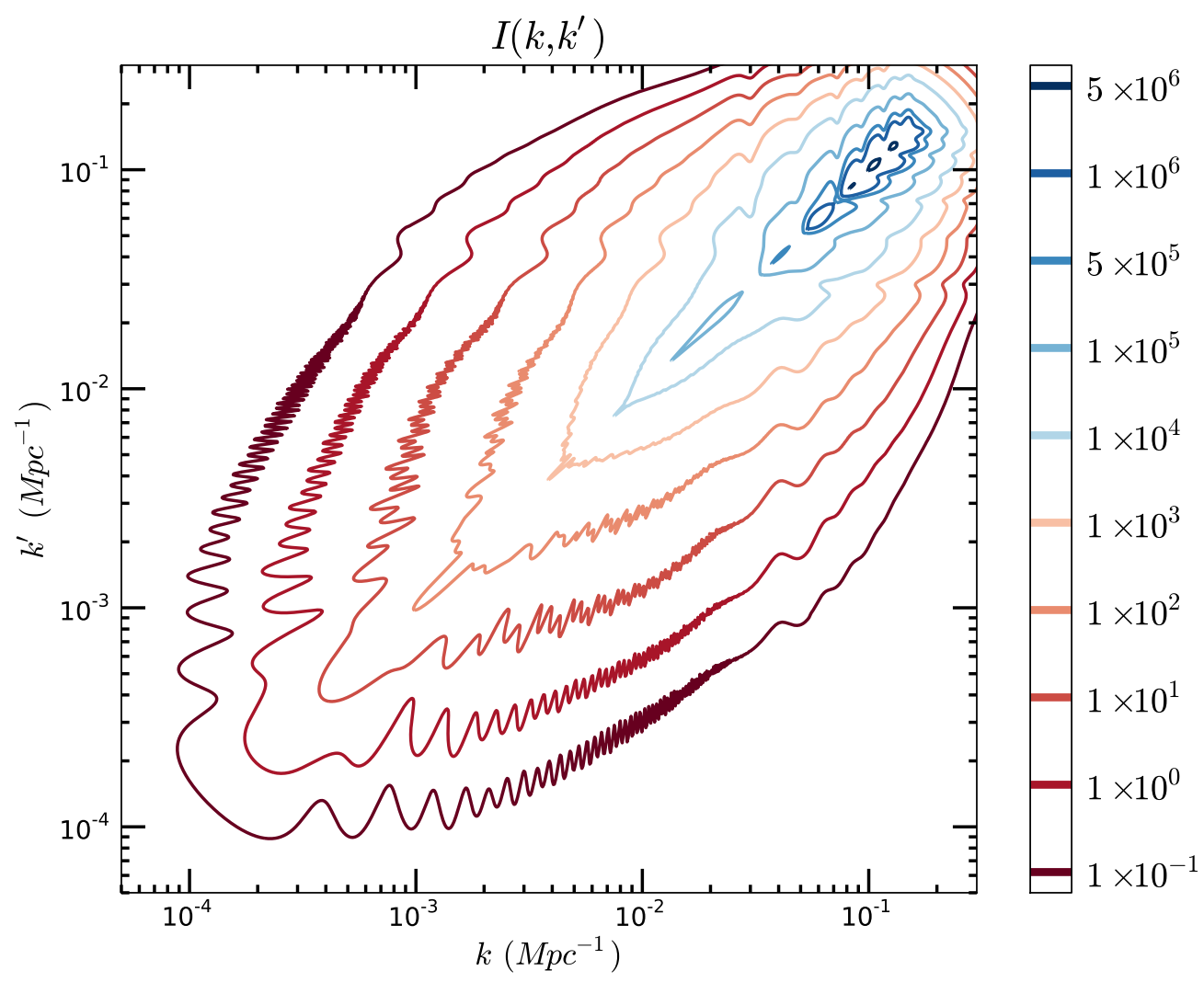

FIG. 1. Fisher information density relative to a reference primordial power spectrum. The information density defined in eqn. (6) is shown.

The full-sky temperature power spectrum $C_{\ell}$ depends linearly on the primordial power spectrum and is defined as the following linear integral transform

$$
C_{\ell}^{T T}=\int_{-\infty}^{\infty} d \ln k P(k)\left[\Delta_{\ell}^{(T)}(k)\right]^{2}
$$

where $\Delta_{\ell}^{(T)}(k)$ is an adiabatic temperature transfer function as computed using standard Boltzmann solver such as CAMB [35]. The polarization and cross spectra, $C_{\ell}^{E E}$ and $C_{\ell}^{T E}$, are similarly defined. We approximate the likelihood by assuming full sky coverage and uncorrelated white noise

$$
-\ln L=\frac{f_{\text {sky }}}{2} \sum_{\ell=2}^{\ell_{\max }}(2 \ell+1)\left[\operatorname{tr}\left(\frac{\mathbf{C}_{\ell}^{(o b s)}}{\mathbf{C}_{\ell}^{(t h)}+\mathbf{N}_{\ell}}\right)-\ln \operatorname{det}\left(\frac{\mathbf{C}_{\ell}^{(o b s)}}{\mathbf{C}_{\ell}^{(t h)}+\mathbf{N}_{\ell}}\right)-3\right]
$$

where $\mathbf{C}_{\ell}^{(o b s)}=\left\{C_{\ell}^{(o b s), A B}\right\}_{A, B=T, E}$ is the measured power spectrum matrix, $\mathbf{C}_{\ell}^{(t h)}$ the theoretical (predicted) power spectrum matrix, and $\mathbf{N}_{\ell}$ is the detector noise. In this section 

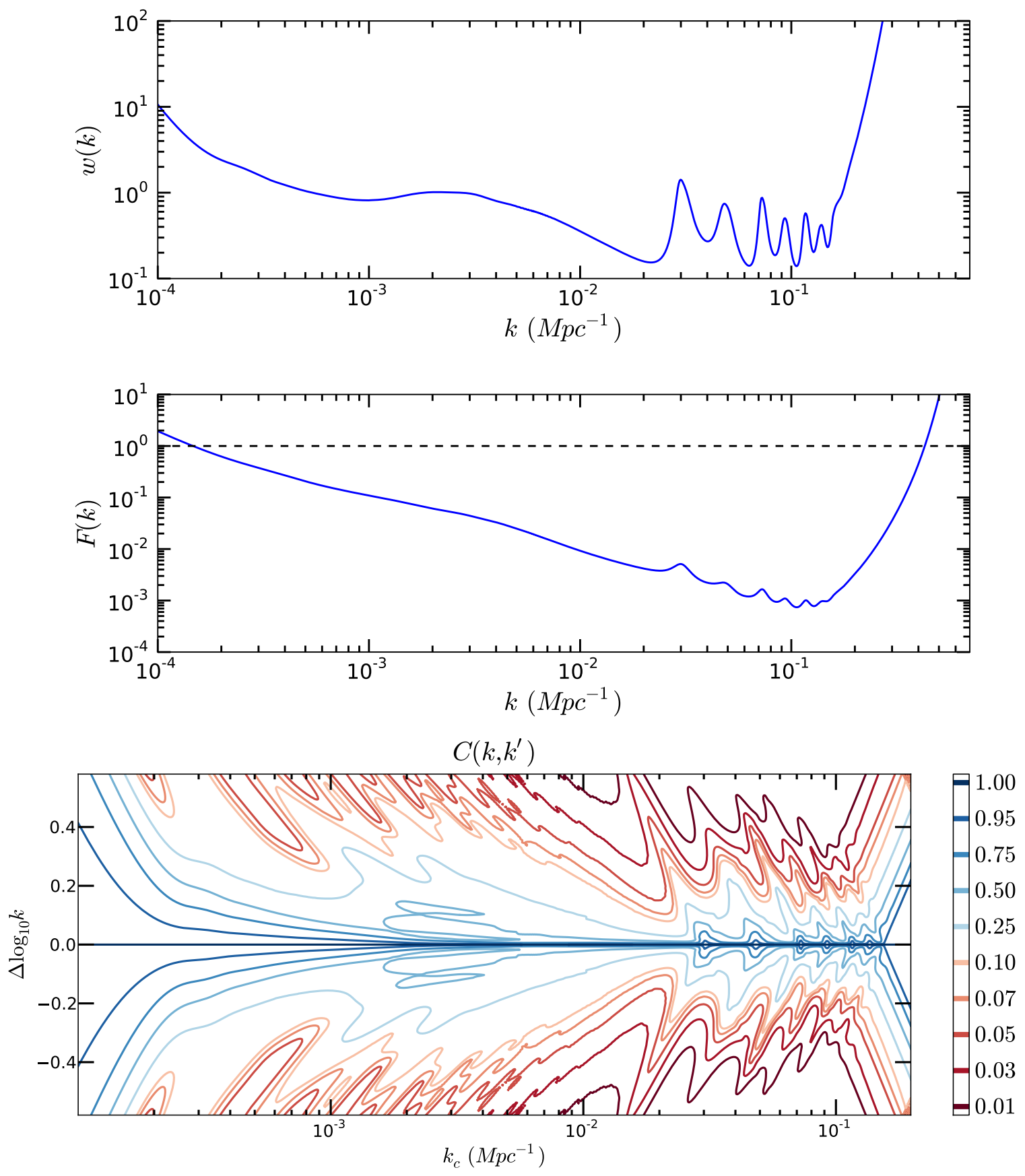

FIG. 2. Properties of the Fisher information density kernel. The top plot shows the width as defined in eqn. (7). The middle plot shows $F(k)$ as defined in eqn. (9), which provides an estimate of how accurately fluctuations at the scale $k$ are being constrained. The bottom plot shows the structure of the correlation matrix (i.e., the kernel $\left.C\left(k, k^{\prime}\right)=I\left(k, k^{\prime}\right) /\left[I(k, k) I\left(k^{\prime} k^{\prime}\right)\right]^{1 / 2}\right)$. Here horizontal coordinate is $k_{h o r}=\sqrt{k k^{\prime}}$ and the vertical coordinate is $\ln \left(k^{\prime} / k\right)$. On the far left and the far right the elements far away from the diagonal have a correlation of almost one, indicating that only a single quantity is being measured at very small and very large $k$.

and elsewhere in the paper unless otherwise specified, we assume a fictitious experiment 
somewhat similar to Planck based on the specifications published in the bluebook [2] rather than on an updated understanding of the mission and the instrument in-flight performance.

In this paper we are concerned with the effect that small scale variations in the primordial power spectrum have on the likelihood. We define the Fisher information kernel $I$ as

$$
-2 \ln L=\int d \ln k \int d \ln k^{\prime}\left[P(k)-P_{M L}(k)\right] I\left(k, k^{\prime}\right)\left[P\left(k^{\prime}\right)-P_{M L}\left(k^{\prime}\right)\right]
$$

where an irrelevant constant term has been omitted. Using this definition we can express the kernel as a functional derivative of the log likelihood

$$
I\left(\ln k, \ln k^{\prime}\right)=\left.\frac{\delta^{2}(-\ln L)}{\delta P(\ln k) \delta P\left(\ln k^{\prime}\right)}\right|_{P=P_{M L}}
$$

where $P_{M L}$ is the primordial power spectrum at maximum likelihood.

Since we already have a rough idea of what the primordial power spectrum looks like on the scales probed by the $\mathrm{CMB}$, it makes sense to describe the power spectra under consideration in terms of fractional variations with respect to a fiducial power law spectrum (for which the parameters are taken from the WMAP 7-year best fit model). We set $P_{0}(k)=$ $A\left(k / k_{0}\right)^{n_{S}-1}$ where $A=2.42 \times 10^{-9}, k_{0}=2.0 \times 10^{-3} \mathrm{Mpc}^{-1}$, and $n_{S}=0.966$, and define $P(k)=P_{0}(k)[1+f(k)]$. Let us for the moment fix the other cosmological parameters to their WMAP 7-year best fit values. We may express the expectation value of the relative log likelihood as the following quadratic form about the best-fit value as follows:

$$
\Delta[-\ln L]=\frac{1}{2} \int d\left(\ln k_{1}\right) \int d\left(\ln k_{2}\right)\left[f\left(k_{1}\right)-f_{M L}\left(k_{1}\right)\right] \hat{I}\left(k_{1}, k_{2}\right)\left[f\left(k_{2}\right)-f_{M L}\left(k_{2}\right)\right]
$$

where $\hat{I}\left(k, k^{\prime}\right)=P_{0}\left(k^{\prime}\right) I\left(k, k^{\prime}\right) P_{0}(k)$. In our definitions we have tried to remove dimensions from quantities wherever possible. Fig. 1 shows the Fisher information density $\hat{I}\left(k_{1}, k_{2}\right)$. The integral over both arguments is interpreted as the $\chi^{2}$ for detecting a CMB signal over a null hypothesis of no CMB signal at all, and as expected this quantity is approximately $10^{6}$ or $\ell_{\max }^{2}$ where $\ell_{\max }$ is approximately the multipole number where the signal-to-noise ratio $(S / N)$ has dropped to one. Were it not for correlations in the Fisher information density, we would be able to measure about a million independent quantities characterizing the shape of the power spectrum at a marginal statistical significance - that is, at about $1 \sigma$. This would indeed be the case if the Fisher information density were more strongly peaked along the diagonal. The width of the peaked region along the diagonal provides an approximate idea of how coarsely one has to bin for correlations between adjacent bins to be 
negligible. If the peaked region along the diagonal is very wide, we measure a small number of quantities (much smaller than $\chi_{\text {total }}^{2}$ ) albeit at very high precision. The most extreme case would be a Fisher matrix where all the entries are equal, or more generally of the form $\hat{I}\left(\kappa_{1}, \kappa_{2}\right)=g\left(\kappa_{1}\right) g\left(\kappa_{2}\right)$ where we define $\kappa=\ln (k)$. In this case the Fisher information operator would be of rank one and precisely one quantity can be being measured. Fig. 2 shows the diagonal of the Fisher information kernel and the correlation matrix obtained from the Fisher information kernel- that is $C\left(\kappa, \kappa^{\prime}\right)=\hat{I}\left(\kappa, \kappa^{\prime}\right) /\left[\hat{I}(\kappa, \kappa) \hat{I}\left(\kappa^{\prime}, \kappa^{\prime}\right)\right]^{1 / 2}$. The fact that at very low $k$ and very high $k$ the correlation becomes very nearly one even quite far from the diagonal indicates that in each of these two region there is only one relevant quantity that is being measured constituting a sort of weighted average of the power spectrum at each end. It is convenient to define the local width of the diagonal in the following way
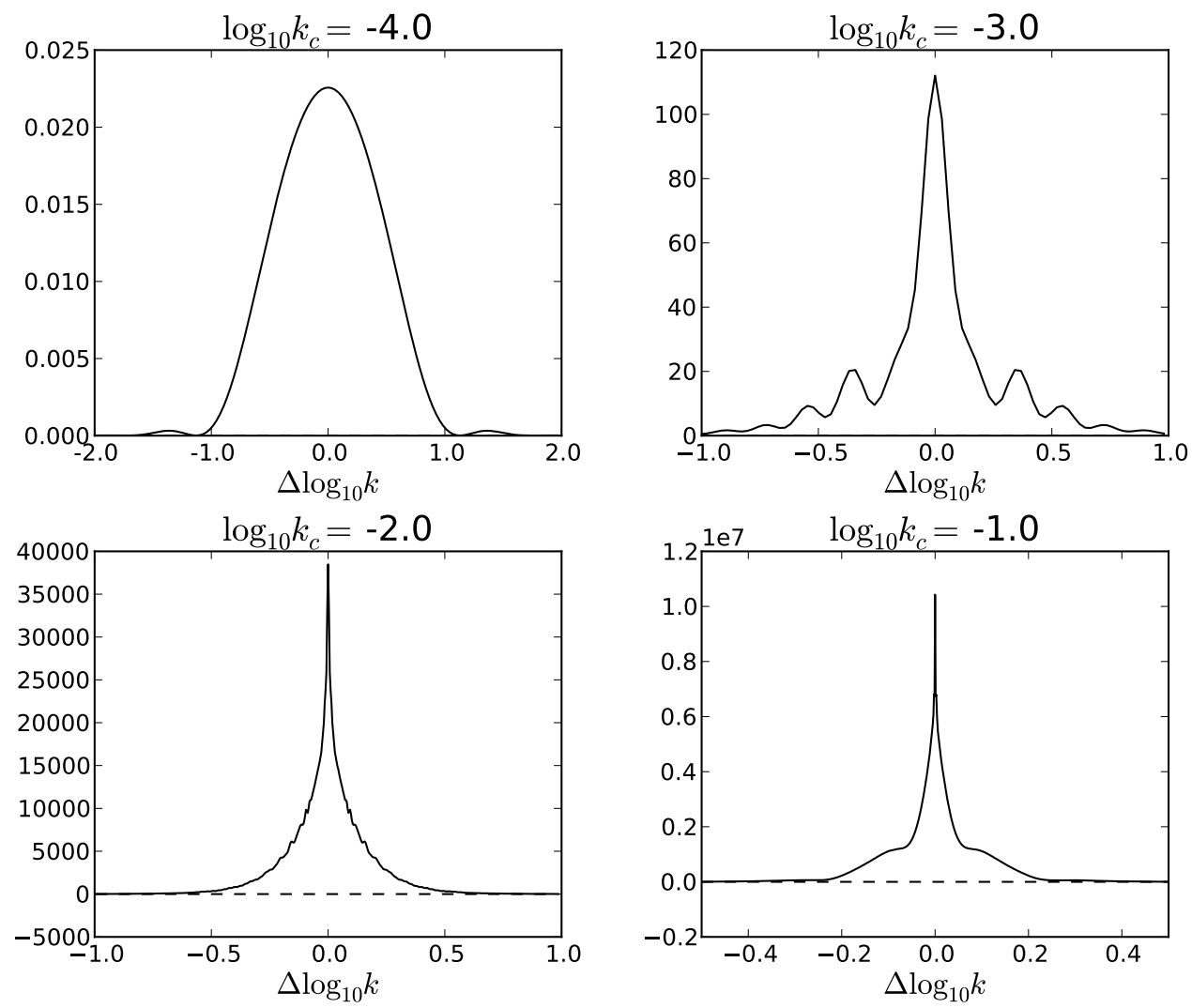

FIG. 3. Fisher density kernel transverse profile. We plot the Fisher density kernel in the direction orthogonal to the diagonal (plots labeled according to wavenumber $k_{c}$ of diagonal crossing). (i.e., $\hat{I}\left(k_{c}+\Delta k, k_{c}-\Delta k\right)$ 


$$
w(\ln k)=\frac{\int d\left(\ln k^{\prime}\right) \hat{I}\left(\ln k, \ln k^{\prime}\right)}{\hat{I}(\ln k, \ln k)} .
$$

This quantity is plotted in Fig. 2 in the middle panel. For $\Delta(\ln k) \gtrsim w$, the error in the binned power spectrum may be approximated as

$$
\sigma\left[\frac{\delta P}{P}_{k \in\left[k_{1}, k_{2}\right]}\right] \approx\left[\hat{I}\left(\frac{1}{2} \ln k_{1} k_{2}, \frac{1}{2} \ln k_{1} k_{2}\right) w\left(\frac{1}{2} \ln k_{1} k_{2}\right) \ln \left(\frac{k_{2}}{k_{1}}\right)\right]^{-1 / 2} .
$$

Fig. 2 shows (on a logarithmic scale)

$$
F(\ln k)=[\hat{I}(\ln k, \ln k) w(\ln k)]^{-1 / 2} .
$$

When $F \gtrsim 1$ we have essentially no useful information regarding the power spectrum given current expectations.

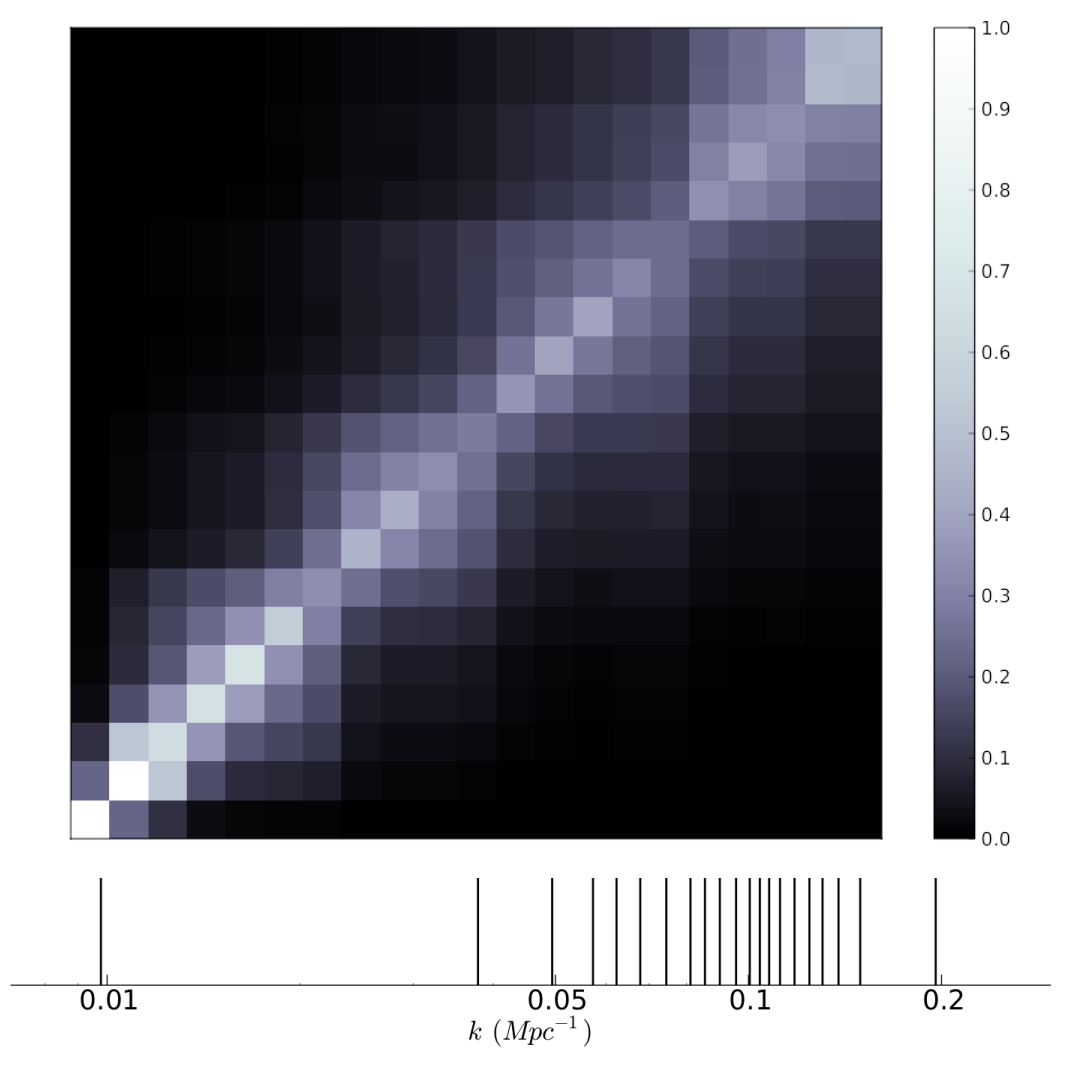

FIG. 4. For the partition into bins indicated at the bottom, the images show the correlations between the bins using a greyscale. 100 bins were chosen so that the information in each bin as defined in the text is approximately equal. Each bin contains a $\chi^{2}$ corresponding to a detection at about $100 \sigma$ of power within that bin.

We may choose bins of varying width so that each bin contains roughly the same amount of information. The bottom of Fig. 4 shows how the domain in wavenumber can be subdivided 
into approximately 100 bins each containing about a detection at $100 \sigma$ (relative to the null hypothesis of a vanishing power spectrum in that bin). The locations of the bin boundaries are shown in the lower panel of the plot. The upper panel of Fig. 4 shows using a greyscale density plot the correlations between bins.

On smaller scales the story is more complicated. The precise profile of the correlations near the diagonal is relevant for determining what information can be extracted concerning small-scale structure in the power spectrum. In Fig. 3 the transverse profile of the Fisher information density kernel is shown for some representative wavenumbers. The shape of the transverse profile is key to determining how much information is available for reconstructing variations in the primordial power spectrum that change rapidly with $k$. A smooth broad profile acts to erase information on small scales in $k$ whereas a sharp narrow profile facilitates recovering information concerning such rapid variations.

Relative to a given experiment or collection of datasets, over what range in $k$ are the parameters of the power spectrum, here $n_{S}$ and $\beta$, actually being measured? This is an important question because the parametric model serves more as a local fitting function for the power spectrum and does not derive from a fundamental theory allowing us to shamelessly extrapolate the power law as far as we want without trepidation. If we really knew that our power law ansatz were correct, this would not be a relevant question. We would like to define $\kappa^{\text {low }}$ and $\kappa^{\text {high }}$ to characterize how far to the left and to the right the bulk of the statistical information extends. Given the parameterization for the power spectrum

$$
P(k)=A\left(k / k_{0}\right)^{\left(n_{S}+\beta \ln \left(k / k_{0}\right)\right)},
$$

it follows that

$$
\ln P(k)=\ln A+n_{S} \ln \left(k / k_{0}\right)+\beta \ln ^{2}\left(k / k_{0}\right)
$$

and to lowest order

$$
\frac{\delta P}{P_{0}}=\frac{\delta A}{A}+\left(\delta n_{S}\right)\left(\kappa-\kappa_{0}\right)+(\delta \beta)\left(\kappa-\kappa_{0}\right)^{2}
$$

where $\kappa=\ln k, \kappa_{0}=\ln k_{0}$, and $k_{0}$ is the so-called pivot or reference scale. Under a change of pivot scale $\kappa_{0} \rightarrow \hat{\kappa}_{0}$, the parameters transform as

$$
\begin{aligned}
A\left(\kappa_{0}\right) & \rightarrow A\left(\hat{\kappa}_{0}\right)=\exp \left[\ln A\left(\kappa_{0}\right)+n_{S}\left(\kappa_{0}\right)\left(\hat{\kappa}-\kappa_{0}\right)+\beta\left(\kappa_{0}\right)\left(\hat{\kappa}_{0}-\kappa_{0}\right)^{2}\right], \\
n_{S}\left(\kappa_{0}\right) & \rightarrow n_{S}\left(\hat{\kappa}_{0}\right)=n_{S}\left(\kappa_{0}\right)+\beta\left(\kappa_{0}\right)\left(\hat{\kappa}_{0}-\kappa_{0}\right),
\end{aligned}
$$




$$
\beta\left(\kappa_{0}\right) \rightarrow \beta\left(\hat{\kappa}_{0}\right)=\beta\left(\kappa_{0}\right)
$$

where $\hat{\kappa}_{0}=\ln \hat{k}_{0}$ and $\kappa_{0}=\ln k_{0}$. It is sensible to choose $k_{0}$ so that the uncertainties in $A$ and $n_{S}$ are uncorrelated. Otherwise, because of a lever arm effect, the errors become large and highly correlated. Alternatively, the lack of correlation between $n_{S}$ and $\beta$ could have been chosen to set $\kappa_{0}$, but we have only one free parameter to adjust, and both conditions place $k_{0}$ within what may be described as the 'center of mass' of the statistical information concerning the form of the primordial power spectrum for a specific experiment or a collection of datasets combining multiple experiments.

Having chosen a sensible pivot scale, we first consider a simplified situation with a diagonal Fisher information density for the power spectrum so that

$$
\chi^{2}=\int d \kappa I_{\text {diag }}(\kappa)\left[P_{\text {model }}(\kappa)-P_{\text {data }}(\kappa)\right]^{2} .
$$

In this situation, the condition fixing the pivot scale is

$$
\int d \kappa I_{\text {diag }}(\kappa)\left(\kappa-\kappa_{0}\right)=0
$$

and the unmarginalized information (inverse of the variance of the measurement with the other parameters fixed) $n_{S}$ and $\beta$ are given by the two integrals

$$
I_{n_{S}}=\int d \kappa I_{\text {diag }}(\kappa)\left(\kappa-\kappa_{0}\right)^{2}
$$

and

$$
I_{\beta}=\int d \kappa I_{\text {diag }}(\kappa)\left(\kappa-\kappa_{0}\right)^{4}
$$

For the Fisher density of an actual experiment, there are always correlations even if the information is strongly clustered near the diagonal and the integrals in eqns. (16) and (17) become double integrals. We modify eqns. (16) and (17) by limiting the integration over just one of the two variables of integration. For the information concerning each variable, the cumulative information up to a certain $\hat{\kappa}$ is defined as follows, with the expression for $n_{S}$ given by the integral

$$
I_{n_{S}}(\hat{\kappa})=\int_{-\infty}^{\hat{\kappa}} d \kappa \int_{-\infty}^{+\infty} d \kappa^{\prime} I\left(\kappa-\kappa^{\prime} / 2, \kappa+\kappa^{\prime} / 2\right)\left(\kappa-\kappa_{0}\right)^{2}
$$

and we define $\kappa_{n_{S}}^{\text {low }}$ and $\kappa_{n_{S}}^{\text {high }}$ as the locations of the the first and third quartiles, respectively, so that $I_{n_{S}}\left(\kappa_{n_{S}}^{\text {low }}\right)=(1 / 4) I_{n_{S}}$ and $I_{n_{S}}\left(\kappa_{n_{S}}^{\text {high }}\right)=(3 / 4) I_{n_{S}}$, and $\kappa_{\beta}^{\text {low }}$ and $\kappa_{\beta}^{\text {high }}$ can be defined analogously. 
The shape of the cumulative information for $n_{S}$ and $\beta$ are shown in Fig. 5 as well as the locations of the quartiles. (Note that when the matrix is not concentrated along the diagonal and especially in the presence of negative correlations assigning information to a specific $k$ no longer makes sense and the above definitions become problematic.)
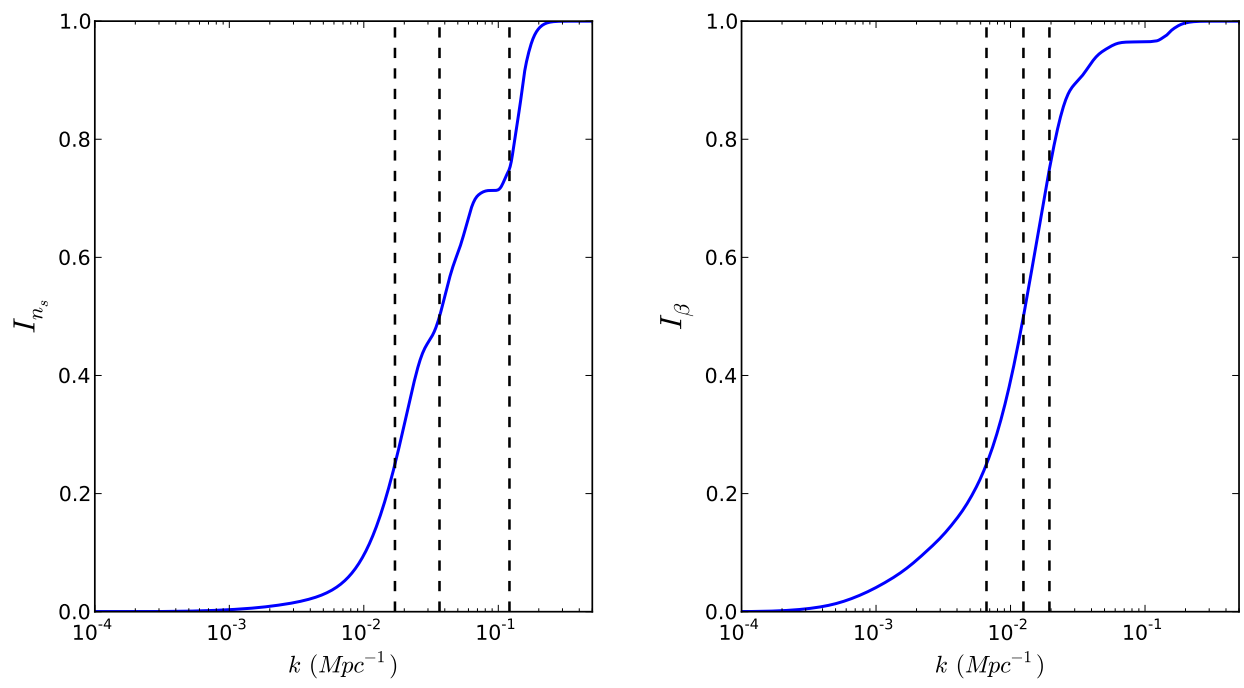

FIG. 5. Cumulative information for $n_{S}$ and $\beta$. The left panel shows the cumulative information for $n_{S}$ as defined in eqn. (18) as well as the first, second, and third quartiles. The right panel shows the analogous information for the running in the spectral index $\beta$. 


\section{POWER SPECTRUM RECONSTRUCTION}

\section{A. Basic approach}

The previous section described the Fisher information density for the fractional variation

$$
f(k)=\delta P(k) / P_{0}(k)
$$

of the primordial power spectrum relative to a reference model taken to be the 7-year WMAP best-fit model [1] already described in the previous section. The Fisher information

density $\hat{I}\left(k, k^{\prime}\right)$ was considered both with the vector $\boldsymbol{\beta}$ containing the other cosmological parameters fixed - that is, assumed to be determined extremely precisely from other nonCMB datasets - and with $\boldsymbol{\beta}$ determined internally using the CMB data alone and hence uncertain. In the latter case we marginalize over the other cosmological parameters treating them as nuisance parameters with a flat (i.e., non-informative) prior probability distribution. In this section we consider how best to reconstruct the power spectrum-or more precisely $f(k)$ - over the range of wavenumbers where the CMB provides sufficient information.

In principle, if the parameters (or perhaps bins) used to describe $f(k)$ are less numerous than the number of independent data points, we could reconstruct $f(k)$ without a regulator, or prior distribution concerning the smoothness of $f(k)$ in the form of a roughness penalty. However since we want to avoid a parametric model as well as artifacts from bin boundaries, we instead introduce a roughness penalty. The Fisher information matrix for the primordial power spectrum takes the general form

$$
I_{f f}=\left(X^{T}\right)_{f c} I_{c c} X_{c f}+\lambda R_{f f}
$$

In the quadratic approximation, minus twice the $\log$ likelihood is given by $\chi^{2}=\mathbf{f}^{T} I_{f f} \mathbf{f}$. Here $I_{c c}$ is Fisher matrix for the angular CMB power spectrum. In order to provide as compact a notation as possible, we write our expressions as if we were dealing with finite-dimensional matrices and vectors, even though the index $f$ represents continuous variable and $c$ is a discrete index. Here $\lambda R_{f f}$ represents a regulator whose eigenvalues should very nearly vanish for slow variations in the power spectrum but be large (relative to the eigenvalues of the Fisher matrix) for modes where $f(k)$ wiggles rapidly. A commonly used but by no means unique choice for this regulator is the quadratic form

$$
\lambda R(f)=\lambda \mathbf{f}^{T} R_{f f} \mathbf{f}=\lambda \int d \kappa\left(\frac{\partial^{2} f(\kappa)}{\partial \kappa^{2}}\right)^{2},
$$


which is a measure of how much the function $f(k)$ deviates from a straight line. Under this regulator, to linear order a change in the amplitude $A_{s}$ or spectral tilt $n_{S}$ suffers no penalty at all, but rapid variations in the running of the spectral index, or a large curvature, are penalized by an amount proportional to the metaparameter $\lambda$. The object is to penalize functions that are too rough through a judicious choice for $\lambda$.

Above we have assumed that the other cosmological parameters, or elements of the vector $\boldsymbol{\beta}$, have been fixed. If instead these are determined internally using the CMB data, the expression for the Fisher information for the primordial power spectrum after marginalization is modified to

$$
I_{f f}^{(\text {marg }, \text { flat })}=\left(X^{T}\right)_{f c}\left[I_{c c}-\left(I_{c c} X_{c \beta}\right)\left(X_{\beta c}^{T} I_{c c} X_{c \beta}\right)^{-1}\left(X_{\beta c}^{T} I_{c c}\right)\right] X_{c f}
$$

and in the presence of additional information on $\boldsymbol{\beta}$ (for example, by incorporating other external non-CMB data sets) expressed by the Fisher matrix $I_{\beta \beta}$, this expression modifies to

$$
I_{f f}^{(\text {marg,prior })}=\left(X^{T}\right)_{f c}\left[I_{c c}-\left(I_{c c} X_{c \beta}\right)\left(X_{\beta c}^{T} I_{c c} X_{c \beta}+I_{\beta \beta}\right)^{-1}\left(X_{\beta c}^{T} I_{c c}\right)\right] X_{c f} .
$$

The equations here assume a Gaussian likelihood as well as Gaussian prior information. The most significant non-Gaussianity arises from the angular power spectrum likelihood at very low $\ell$ and from nonlinearity in the change of the angular power spectrum as a function of variations in the cosmological parameters (i.e., the components of the vector $\boldsymbol{\beta}$ ). Supposing Gaussianity allows simple analytic expressions to be obtained. In practice the distributions are slightly non-Gaussian and must be explored using MCMC or some other numerical sampling method. The Gaussian analysis, however, is invaluable for gaining intuition about how to select a good regulator, but the solutions obtained should subsequently be validated by simulations taking into account the non-Gaussianity of the regulated likelihood.

Fig. 6 6 shows the Fisher density when $h, \omega_{b}$, and $\omega_{c}$ are uncertain and constrained only by the CMB data, and Fig. 7 shows some transverse profiles. These should be compared with the analogous plots in Figs. 1 and 3 of the unmarginalized Fisher density, which are almost identical at small $k$, but have significantly different behavior at high $k$. Marginalization over the cosmological parameters increases the inter- $k$ correlations, as seen in Fig. 8 . In marginalizing over the cosmological parameters, we have not included the reionization optical depth $\tau$, which is doubtless a relevant parameter. This is because except at very low $\ell$, 


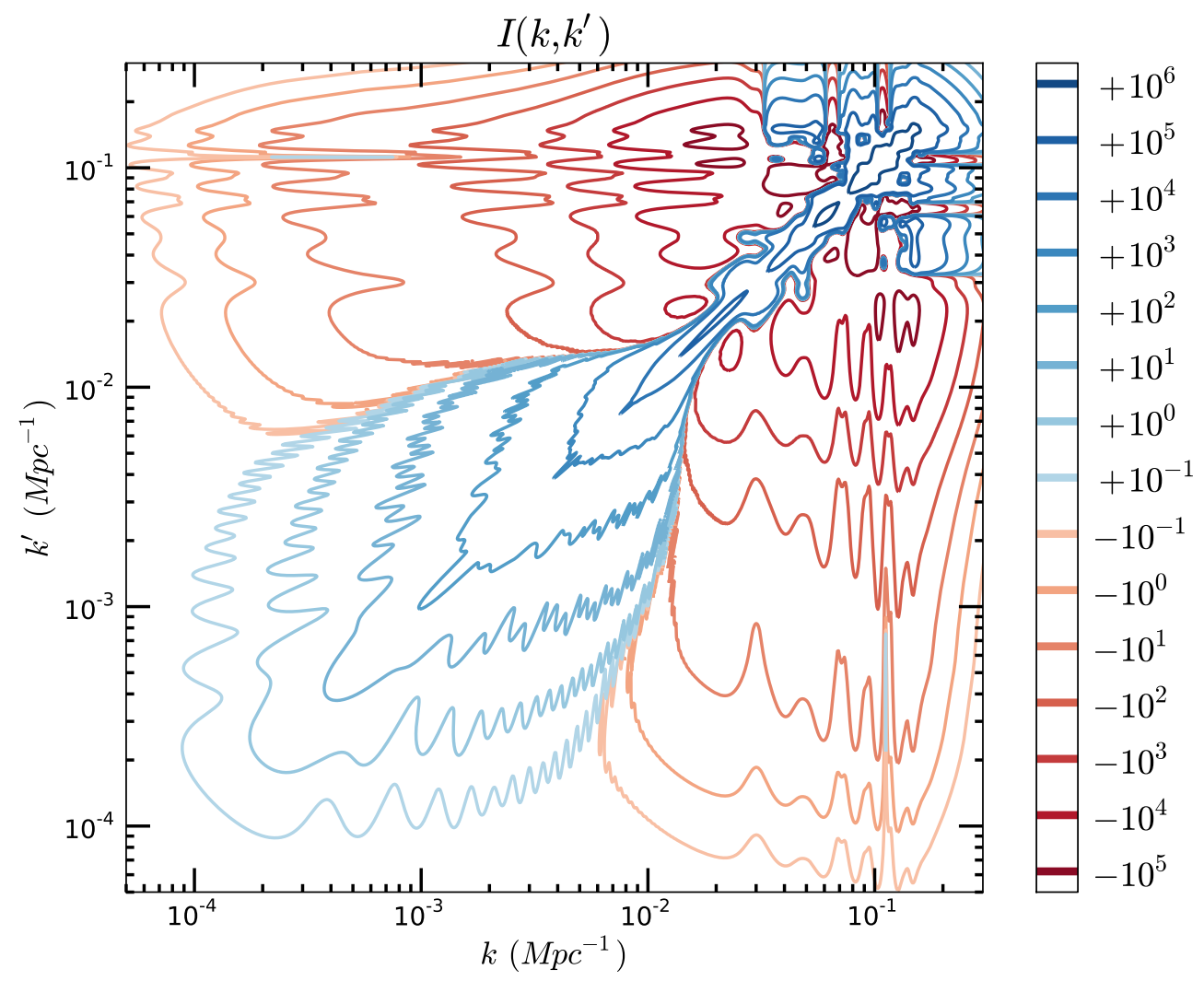

FIG. 6. Fisher density contour plot (after marginalization over the cosmological parameters).

the reionization optical depth $\tau$ and the overall amplitude of the power spectrum $A_{s}$ are completely degenerate. It is the combination $A_{s} e^{-2 \tau}$ that normalizes the overall amplitude but not the shape of the CMB sky power spectrum. Fig. 9 shows the deviations to this statement at low $\ell$. The deviation is greatest at low $\ell$, especially for the E-mode polarization. The data from the very first multipoles can be used to determine $\tau$, and then to determine the overall normalization.

We now return to the problem of choosing the metaparameter $\lambda$ of the regulator. On the one hand, if $\lambda$ is too small, the reconstruction is dominated by noise, seeded by the cosmic variance of a specific sky realization and by random instrument noise on smaller scales. In this situation, the bias of the reconstruction will be small but its variance will be large. On the other hand, if $\lambda$ is too large, real features in the primordial power spectrum, especially those that are sharp or highly localized, will be smoothed over and distorted. This is bias accompanied by very little variance. Finding the right $\lambda$ amounts to making the 

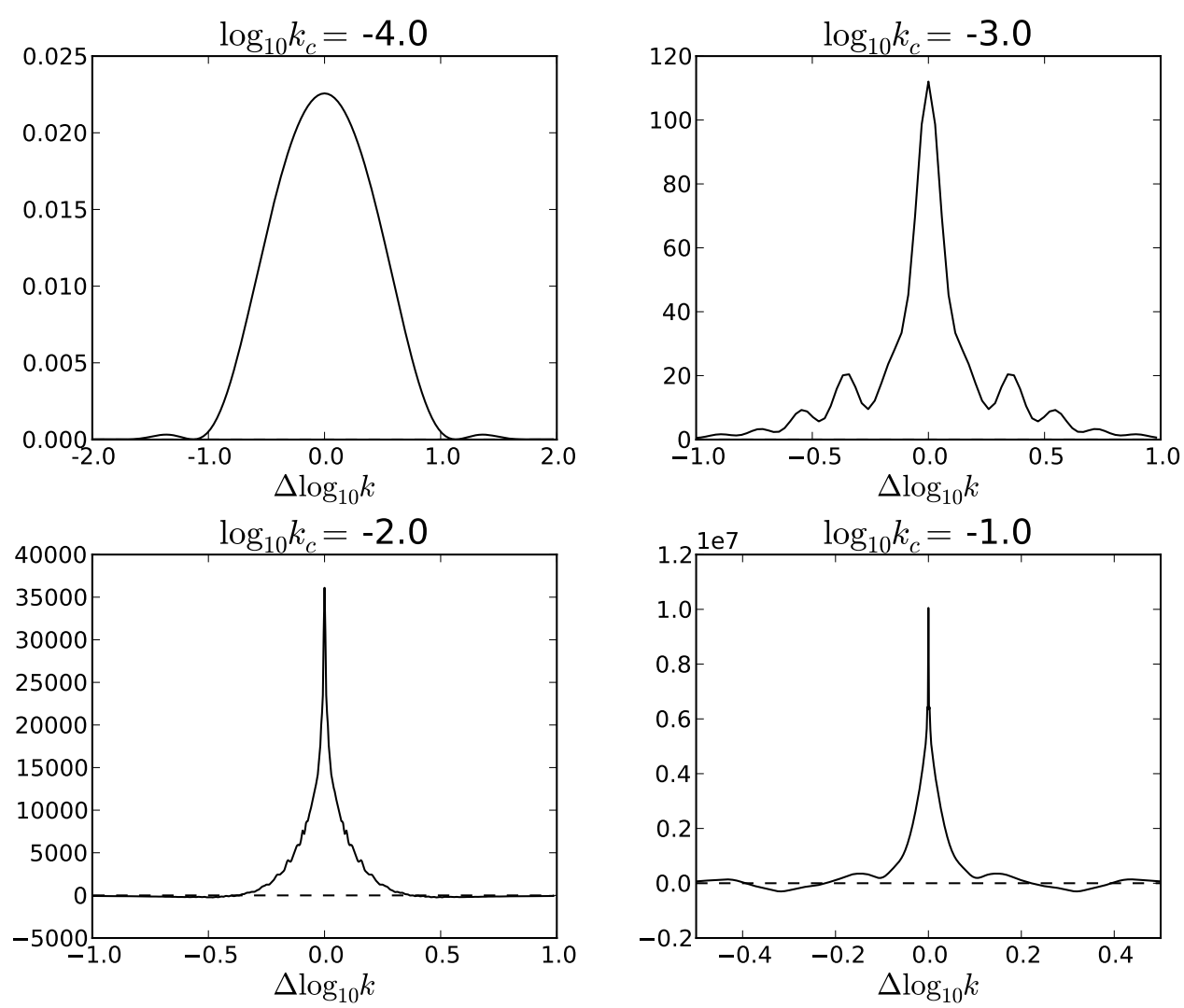

FIG. 7. Cross sections of the Fisher information density along transverse direction (with cosmological parameters uncertain and determined from CMB data alone) centered on the diagonal. The $k_{c}$ indicate the diagonal crossings. Compare these plots to Fig. 3. At large scales $\left(\log _{10} k_{c}=-4\right.$ and $\left.\log _{10} k_{c}=-3\right)$ the kernel is virtually identical in both figures, while at small scales the behavior changes depending on whether the cosmological parameters are fixed or internally determined with uncertainty.

right compromise between bias and variance, both of which should be minimized, resulting in conflicting requirements that cannot be satisfied simultaneously.

The effect of the regulator may be described using the language of Bayesian statistics, as a prior distribution over a function space. While this description provides useful insight, the resulting smoothness prior does not correspond to any actual prior belief concerning the theoretical power spectra. Rather the prior is chosen after the fact and is informed primarily by the characteristics of the experiment and the information provided by the data.

Automatic procedures can be derived for choosing the right parameters for the regulator. In the course of this study we tried using the Aikake Information Criterion (AIC) [13] to choose $\lambda$ as well as criteria penalizing model complexity more strongly inspired by the 


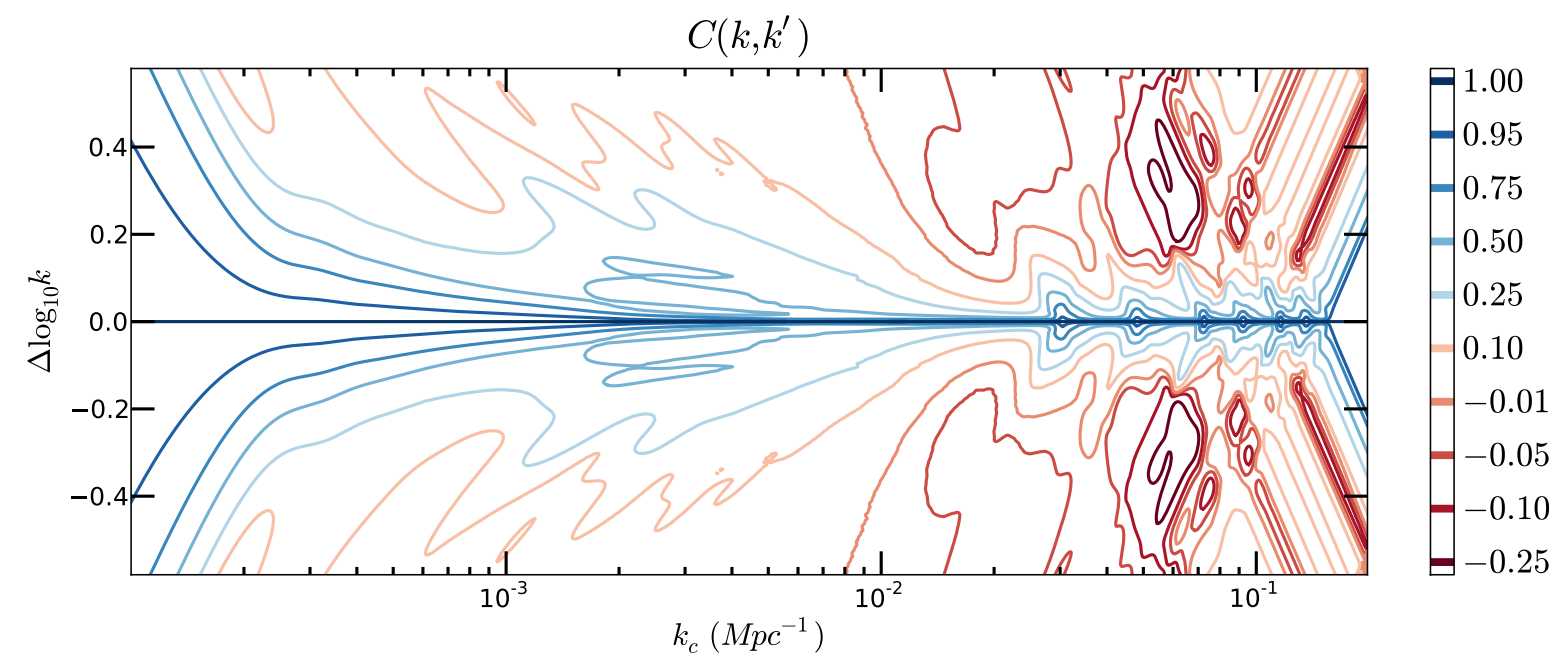

FIG. 8. Correlation after marginalization over the uncertain cosmological parameters determined from CMB alone. Note that there is now much more correlation between very low and very high $k$.

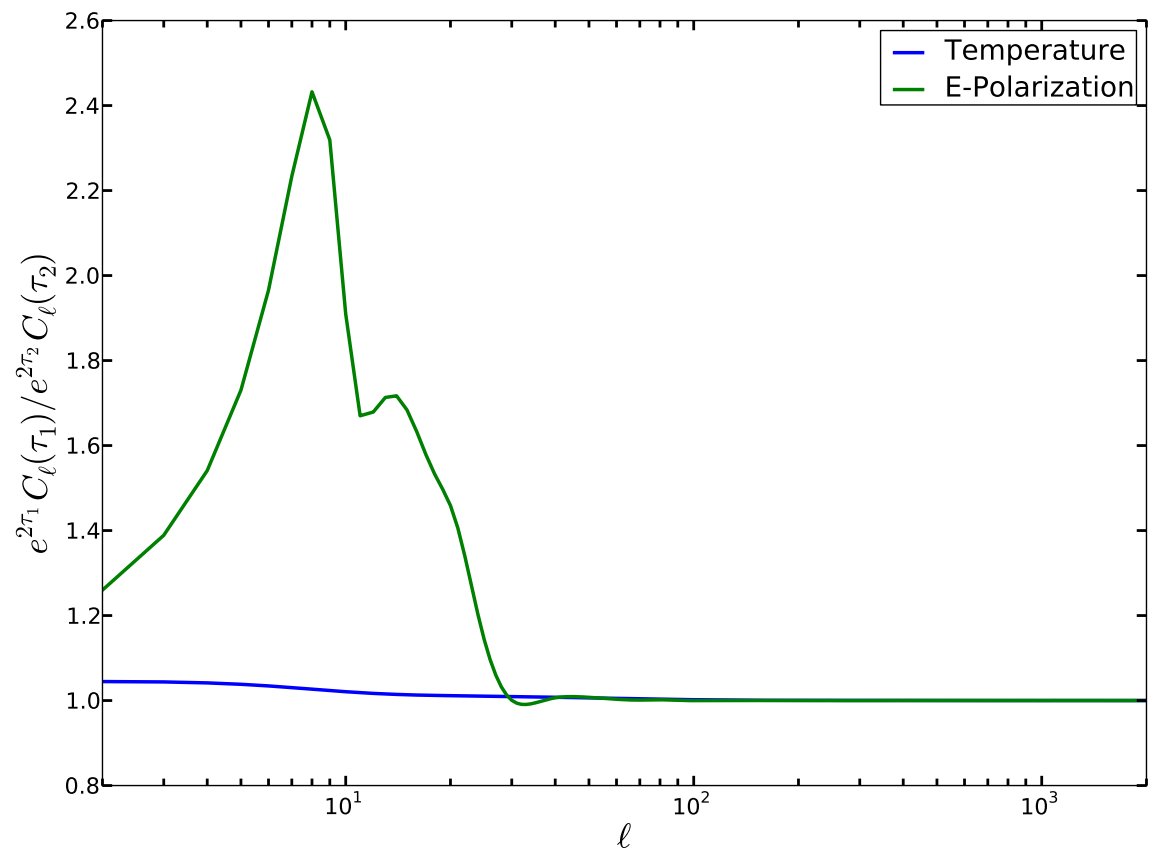

FIG. 9. Ratio of the temperature and E-polarization $C_{\ell}$ 's at two different values of the optical depth: $\tau_{1}=0.1, \tau_{2}=0.08$. For $\ell \gtrsim 30$, the shapes differ by less than $1 \%$, and for $\ell \gtrsim 100$, less than $0.1 \%$.

BIC (Bayesian Information Criterion) [14]. However, while these criteria may offer some theoretical rational for selecting $\lambda$, they do not provide any insight into what kind of features in the power spectrum can and cannot be recovered. 
The problem of choosing a regulator to recover the primordial power spectrum necessarily involves some degree of arbitrariness. The approach adopted here is pragmatic. We construct a family of model features to be reconstructed and then test various regulators with a range of smoothing parameter choices. The final product puts forth a reconstruction procedure whose properties have been well characterized by showing what features can and cannot be recovered given the experiment and at what statistical significance.

The functional form of the regulator adopted in eqn. (21) is somewhat arbitrary. This form could be justified if there were a translation invariance in the variable $\kappa=\ln k$. The discussion of the previous section showed that the CMB provides stringent constraints on $f(k)$ over a window spanning approximately two decades in $k$, but outside this window $f(k)$ is hardly constrained. In fact, for $k$ well below this window - that is, for modes whose wavelengths are superhorizon today - we measure only the local curvature in the geometry and have no way of deducing the precise wavelengths of the long-wavelength modes giving rise to this curvature, which may be approximated within the present horizon using a quadratic approximation. This situation implies that only an integral over the very low- $\ell$ power spectrum is constrained by the CMB data. This fact is evident from the correlation structure of the Fisher matrix at low- $k$ (Figs. 2, 8), which shows that the dimensionless correlation is almost one in the corner. Because of the sharpness of the centrifugal barrier in the differential equation for the spherical Bessel functions, the very low- $k$ power spectrum affects only the quadrupole and has almost no impact on the higher- $\ell$ multipoles. The impact of the high- $\ell$ part of the power spectrum lying above this window is more complicated because at large arguments the spherical Bessel functions oscillate rapidly and fall off slowly according to a power law that does not depend on $\ell$.

This situation suggests that more regulation is needed outside this window than inside, suggesting the following generalization of eqn. (21)

$$
\lambda \mathbf{f}^{T} R_{f f} \mathbf{f}=\int d \kappa \rho(\kappa)\left(\frac{\partial^{2} f(\kappa)}{\partial \kappa^{2}}\right)^{2},
$$

yet this proposal introduces additional arbitrariness in the choice of $\rho$. In order to deal with the region of the power spectrum that is hardly constrained by the CMB data, we found it necessary to modify the regulator by adding a term pushing the reconstructed spectrum toward the fiducial model. Thus the regulator becomes

$$
\mathbf{f}^{T} R_{f f}(\lambda, \alpha) \mathbf{f}=\lambda \int d \kappa\left(\frac{\partial^{2} f(\kappa)}{\partial \kappa^{2}}\right)^{2}+\alpha \int_{-\infty}^{\kappa_{\min }} d \kappa f^{2}(\kappa)+\alpha \int_{\kappa_{\max }}^{+\infty} d \kappa f^{2}(\kappa)
$$


and now has four adjustable parameters. The last two terms constitute an endpoint fixing regulator. Without these terms, the smoothing regulator favors a linear PPS on the far left and the far right where the PPS is almost unconstrained by the CMB data. This behavior leads to spurious deviations from the fiducial model in the reconstructions.

The reconstruction process may be characterized as follows. Let $f_{\text {in }}$ be the input fractional variation of the PPS containing a number of features to be reconstructed. The recovered fractional variation is

$$
f_{\text {recov }}=\bar{f}_{\text {recov }}+\delta f
$$

which is decomposed into a mean component $\bar{f}_{\text {recov }}$ and a random fluctuating component (of vanishing mean) $\delta f$, whose covariance is given by

$$
\langle\delta f \delta f\rangle=(I+R(\lambda, \alpha))^{-1} I(I+R(\lambda, \alpha))^{-1}
$$

and its mean is

$$
\bar{f}_{\text {recov }}=\left[I_{f f}+R_{f f}(\lambda, \alpha)\right]^{-1} I_{f f} f_{\text {in }}=A f_{\text {in }}
$$

The reconstruction operator $A$ is a smoothing operator, which diminishes noise in $f_{\text {in }}$ from cosmic variance and detector fluctuations by averaging or smoothing, but also introduces a bias into the reconstruction $\bar{f}_{\text {recov }}$. This bias is unavoidable since some degree of smoothing is needed to avoid overfitting the data.

For a numerical implementation, we must discretize $f(k)$ by restricting to a finitedimensional function space. We use a function space sufficiently large so that the results are insensitive to the dimension used. In the interest of numerical stability, it is advantageous to reduce the dimensionality of the function space as much as possible. We found that cubic $B$-splines are well suited for avoiding discretization artifacts while keeping the number of control points small. We expand as

$$
f(\kappa)=\sum_{i} f_{i} B^{i}(\kappa)
$$

where $B^{i}(\kappa)$ are cubic $B$-spline basis functions. We work in the continuum limit where the roughness penalty suppresses variations on scales close to the control point spacing. Throughout a grid equally spaced in $\kappa=\ln k$ with $\Delta \kappa=0.01$ is used. 


\section{B. Reconstruction bias with other cosmological parameters fixed}

In this subsection we examine the mean reconstruction error when the other cosmological parameters (i.e. $H_{0}, \omega_{b}, \omega_{c}, \tau$, etc.) are fixed. In the next subsection we generalize taking into account uncertainties in these parameters and the following section examines the random error and questions of statistical significance. Fig. 10 plots the impulse (i.e., $\delta$-function input) response of $A$ without marginalizing over the cosmological parameters. The action of the reconstruction operator $A$ on an impulse spreads a sharp localized input feature. Mathematically this is due to the smoothing regulator, which smears the variations in the power spectrum where its second derivative is large.

The reconstruction operator introduces a bias in the reconstructed signal that underestimates the height of features. Since the process is linear, a full description of the bias takes the form of the two-dimensional kernel, of which some representative cross sections are shown in Fig. 10. However two quantities provide a succinct partial characterization of the kernel: the impulse response width, defined as the standard deviation or square root of the second moment about the mean as a function of $k_{i n}$; and the area under the curve, whose deviation from one indicates how much the amplitude of broad features is distorted.

The limits $\kappa_{\text {low }}$ and $\kappa_{\text {high }}$ of the endpoint fixing regulator in eqn. (25), beyond which the spectrum is effectively constrained to coincide with the fiducial model, single out an interval of $k$ space where to search for features. A concern is that deviations from the fiducial spectrum beyond these limits (where we cannot measure the power spectrum) could result in spurious features inside the free interval. We found that this not to be a problem when the other cosmological parameters were fixed. Several of the plots in Fig. 10 show the impulse response where the input is located in the fixed regions and we observe that except for some small artifacts near the boundary of a small integrated area, there are no artifacts in the interior of the free region.

\section{Reconstruction bias with uncertainty in cosmological parameters}

The situation studied in the previous subsection where the other cosmological parameters not associated with the PPS are assumed known with absolute precision is idealized. In practice, whether these parameters are determined internally from the CMB data alone or 

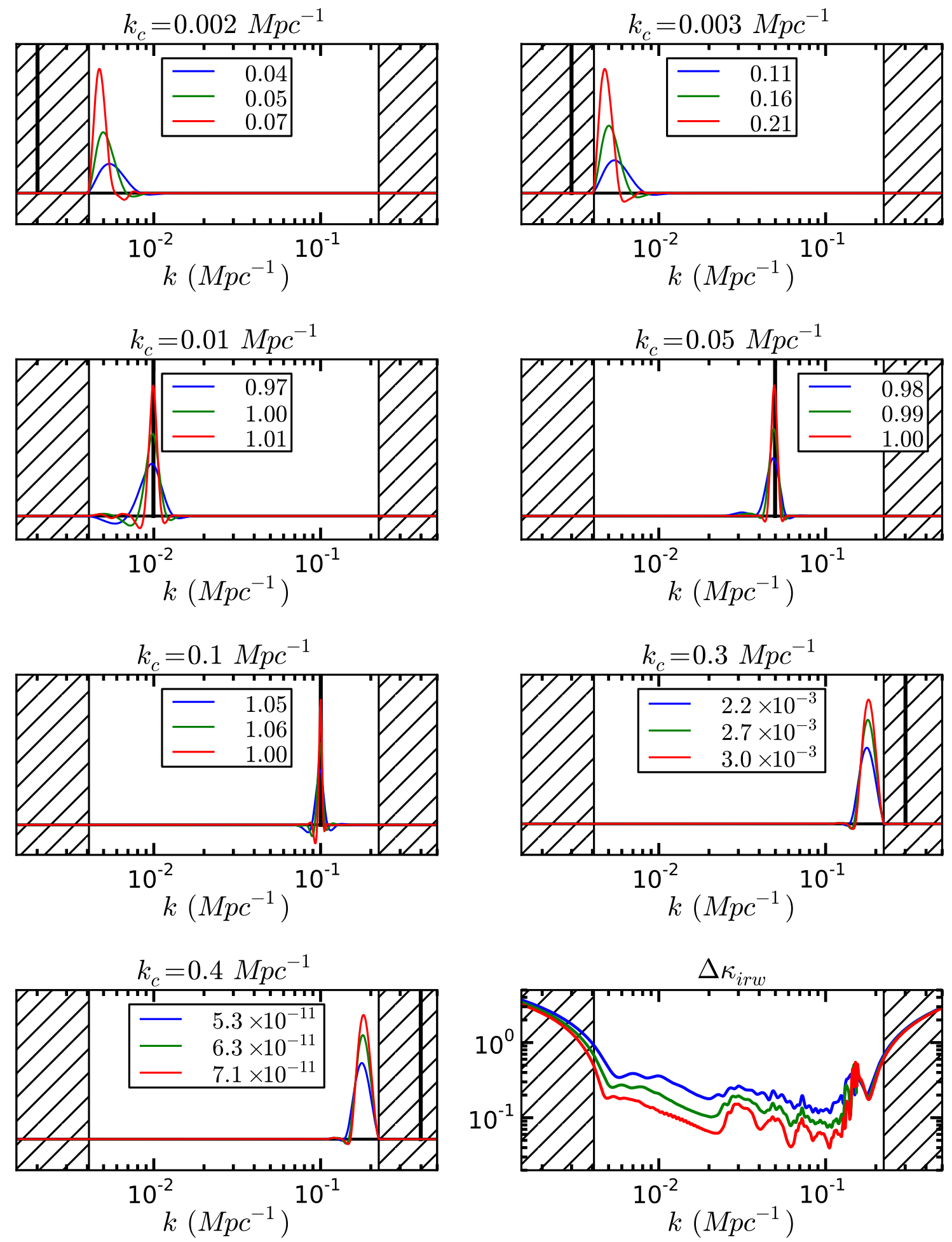

FIG. 10. Impulse response of reconstruction operator $A$ (case with other cosmological parameters fixed). The curves correspond to $\lambda=10^{4}$ (blue), $\lambda=10^{3}$ (green) and $\lambda=10^{2}$ (red). In the shaded regions on the far left and right (where $\kappa<\kappa_{\text {low }}=-5.5$ or $\kappa>\kappa_{\text {high }}=-1.5$ ) an endpoint fixing prior (with $\alpha=10^{4}$ ) has been applied, pushing the reconstruction $f$ to zero there. The numbers in the boxes indicate the area under the curves of the reconstruction kernel, which ideally should be as close as possible to one, the area under the delta function input. The values in the boxes in the two last plots show that there is little leakage from variations in the shaded regions. The lower right plot shows the impulse response width as a function of feature position. 
whether additional non-CMB data is also used (acting as a prior on these parameters), some uncertainty in these additional parameters is always present. This uncertainty degrades the quality of the power spectrum reconstruction, because variations in the power spectrum can be traded against changes in these parameters. This subsection describes the properties of the mean reconstruction in the presence of these uncertainties, with and without external information. The next section treats the noise properties of the reconstruction.

We found that for the case where the other cosmological parameters were determined from the CMB data alone, in order to prevent possible features in the fixed region from introducing spurious features in the free region and spurious shifts in the cosmological parameters, it is necessary to choose a wide interval $\left[\kappa_{\text {low }}, \kappa_{\text {high }}\right]=\left[10^{-4}, 0.3\right] \mathrm{Mpc}^{-1}$ for the free region. $k_{\text {low }}$ here corresponds roughly to the current horizon scale $\left(k_{\text {low }} \sim 1 / \eta_{0}\right.$ where $\eta_{0}$ is the distance to the last scattering surface). $k_{h i g h}$ is slightly above the $k$ corresponding to $\ell_{\max }$. The situation contrasts with the case of the previous subsection where the cosmological parameters are fixed, for which $\left[\kappa_{\text {low }}, \kappa_{\text {high }}\right]$ can be chosen freely without introducing artifacts. For the remainder of the paper we set $\alpha=10^{4}$ and define the free region as $\left[10^{-4}, 0.3\right] \mathrm{Mpc}^{-1}$.

The top row of Fig. 11 shows that the reconstruction operator badly distorts an infinitely narrow impulse input in the case where $h, \omega_{b}$, and $\omega_{c}$ are uncertain and constrained only by the CMB data. This distortion is due to trading variations in the cosmological parameters against variations in the power spectrum, leading to a reconstruction kernel having long oscillating tails in the absence of additional non-CMB information, as in the case plotted here. Fig. 11 also shows the response to a Gaussian input profile of varying width as plotted in the bottom two rows. The bad behavior in the tails of the reconstruction kernel almost disappears when the kernel is applied to a broadened input. Fig. 12 shows that in the region of $k$-space where the bias is most extreme, the response of an impulse away from the central peak oscillates rapidly as the location $k_{c}$ of the input impulse is varied. Although the amplitude of the response in the tail oscillates, its shape remains roughly constant as shown in the figure. This is why the oscillations in the tail interfere destructively almost canceling each other when the impulse input is replaced with a broadened Gaussian input.

Allowing for uncertainty in $h, \omega_{c}$ and $\omega_{b}$, not only modifies the reconstruction kernel, but also causes features in the power spectrum to displace the maximum likelihood cosmological parameters. In the absence of a regulator the mean of this effect would vanish, but the mean is nonzero for $\lambda>0$. By expanding the transfer function to first order around the 

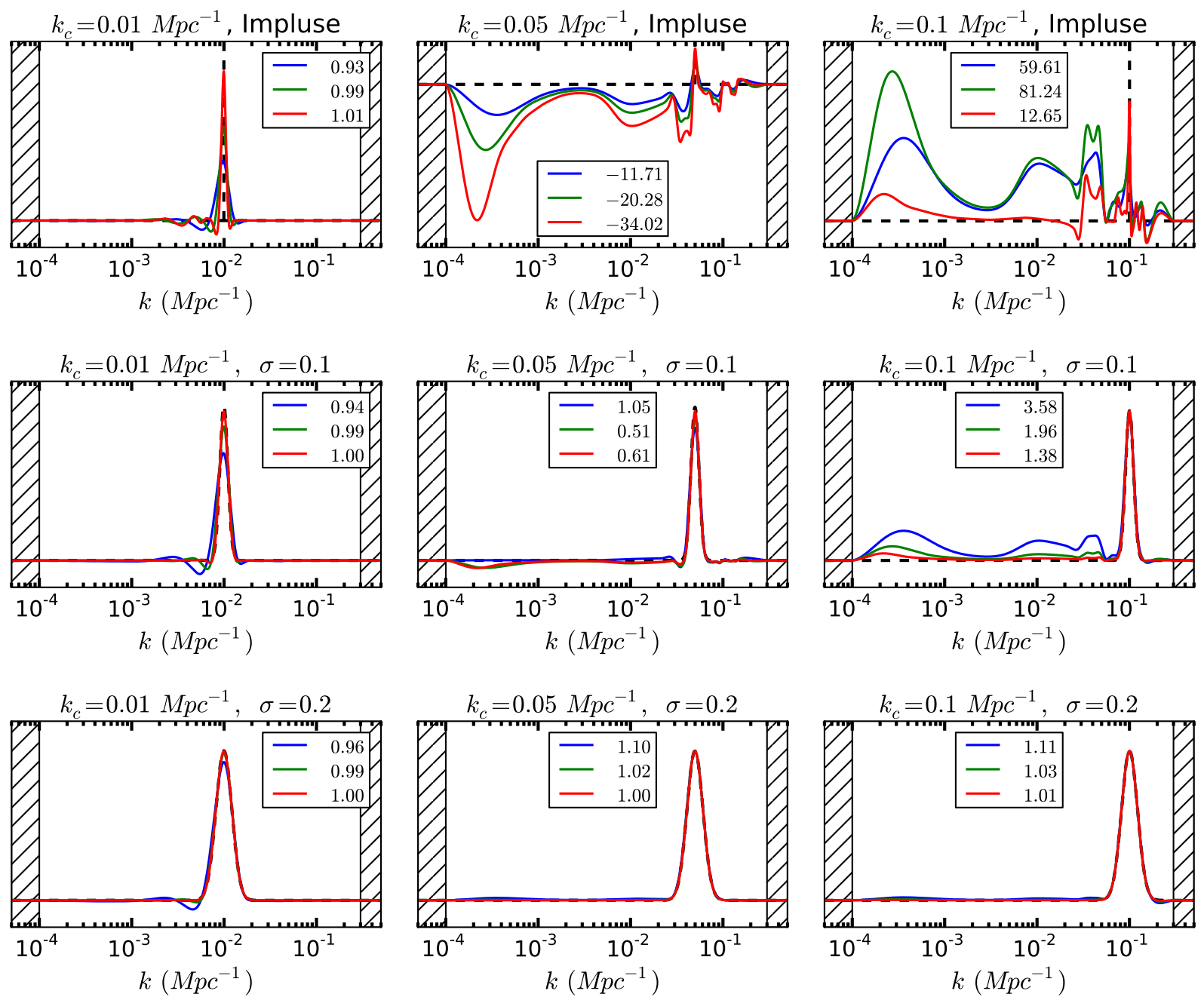

FIG. 11. Smoothing Response to impulse and Gaussian inputs (with marginalization over the other cosmological parameters). The values of the prior parameters and their color mapping are the same as in Fig. 10. The endpoint fixing regulator limits here are $k_{\text {low }}=10^{-4} \mathrm{Mpc}^{-1}$ and $k_{\text {high }}=0.3 \mathrm{Mpc}^{-1}$.

input cosmological parameters, we can express the change in the $C_{\ell}$ 's as a linear function of the change in the PPS and the change in the cosmological parameters $\delta \boldsymbol{\beta}$ :

$$
\delta C_{\ell}=T_{c f} f+T_{c \beta} \delta \boldsymbol{\beta} .
$$

If we treat $(f, \delta \boldsymbol{\beta})$ as a single parameter vector, we can write an extended reconstruction operator $A$ that acts on the space of $(f, \delta \boldsymbol{\beta})$, which we can write in block form as

$$
A=\left[\begin{array}{ll}
A_{f f} & A_{f \beta} \\
A_{\beta f} & A_{\beta \beta}
\end{array}\right]
$$



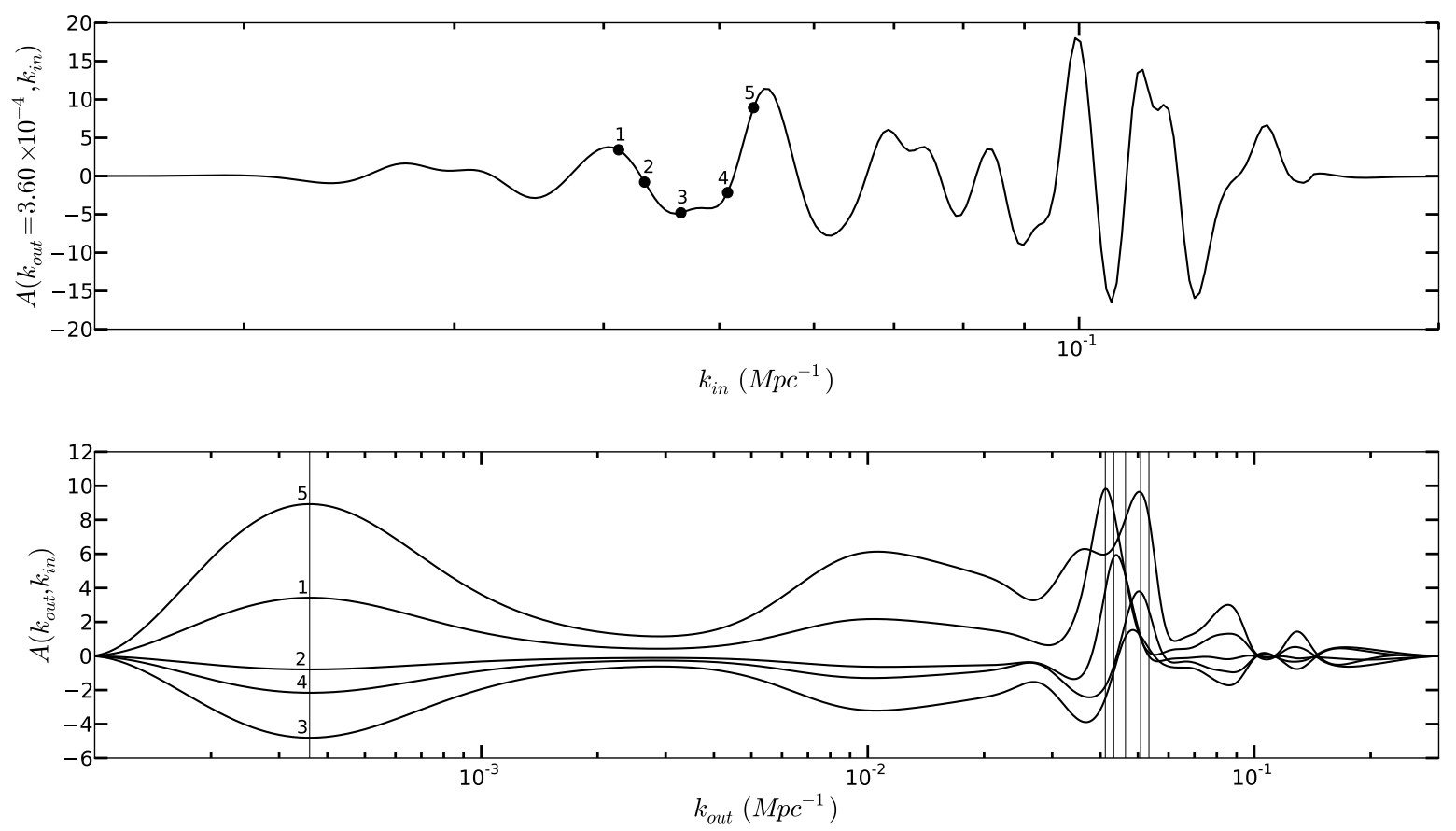

FIG. 12. Rapid oscillations in the marginalized smoothing operator $A\left(k_{\text {out }}, k_{i n}\right)$. The bottom plot shows $A\left(k_{\text {out }}, k_{\text {in }}\right)$ versus $k_{\text {out }}$ for different $k_{\text {in }}$. The black vertical bars on the right indicate five values of $k_{i n}$ for which $A\left(k_{\text {out }}, k_{i n}\right)$ is plotted. We observe that the shape of the tail of the kernel remains approximately fixed but its overall amplitude undergoes oscillations as $k_{i n}$ is varied. The top figure shows $A\left(k_{\text {out }}, k_{i n}\right)$ as a function of $k_{i n}$ with $k_{\text {out }}$ fixed at the value of $k$ corresponding to the position of the single vertical line on the left in the bottom plot. The points show the $k_{i n}$ values for the curves of the bottom plot.

$A$ is defined by inserting $\hat{I}$ from eqns. 222 or 23 into eqn. (28). Due to the off-diagonal block $A_{\beta f}$, a feature in the PPS will in general shift the values of the cosmological parameters.

For small $\lambda$ it is useful to include other data to prevent the other parameters from taking unreasonable values. The constraints from external data used here are $h=0.72 \pm 0.08$ from [36] (HST key project) and $\Omega_{b} h^{2}=(2.2 \pm 0.1) \times 10^{-2}$ from [37, 38] (measured deuterium abundances), but $\Omega_{c} h^{2}$ is left unconstrained by the external data. These constraints are implemented as Gaussian priors in our calculations. Fig. 13 shows that in the absence of external data, for small values of $\lambda$ the determination of the cosmological parameters degrades considerably, contradicting what is known from external data. With external data constraining $h$ and $\omega_{b}$ the situation is remedied substantially, although for interesting values of $\lambda$, up to $20 \%$ errors in $\Omega_{c} h^{2}$ persist.

With external datasets included, the deformation of a signal due to the reconstruction operator is lessened. In Fig. 14, the impulse and Gaussian response of the reconstruction 


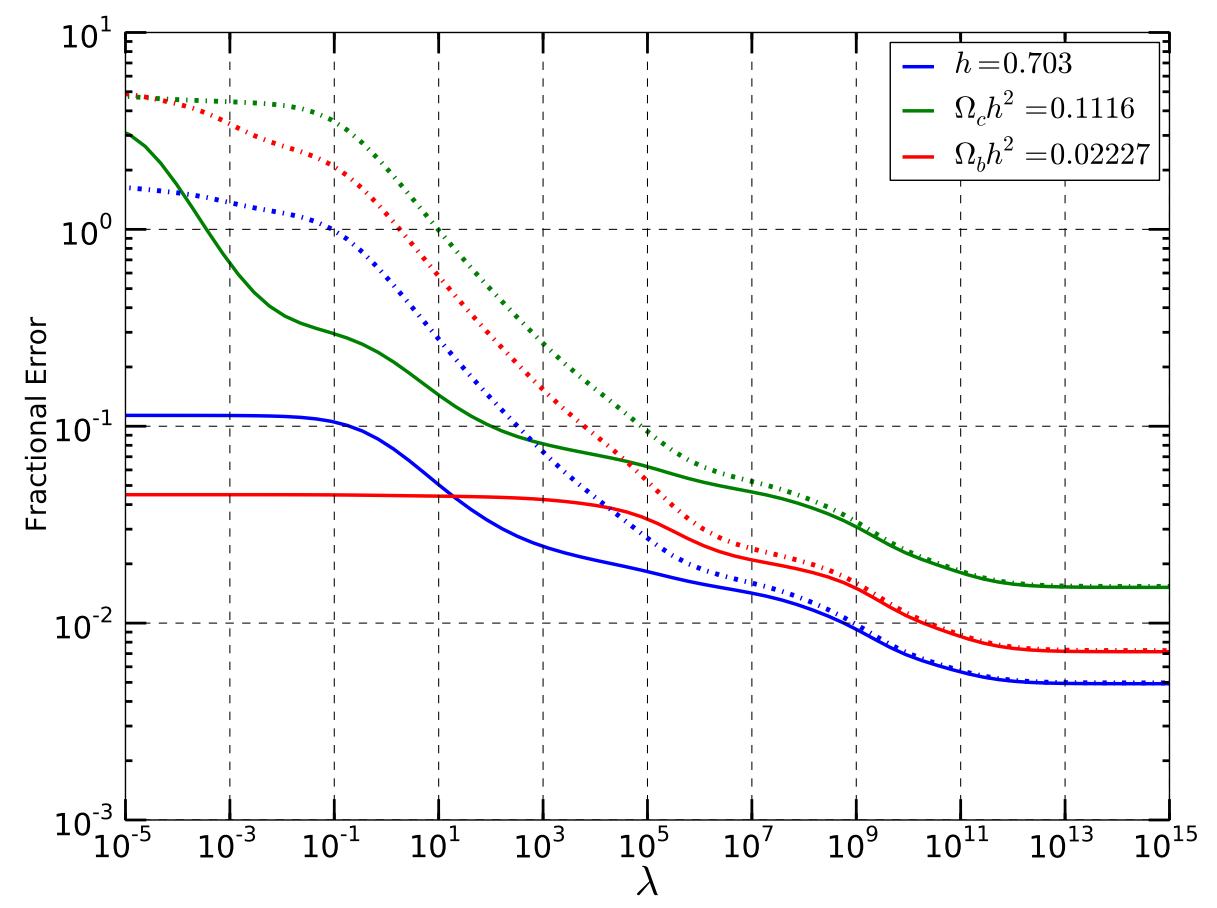

FIG. 13. Fractional error in cosmological parameters as a function of the smoothing parameter $\lambda$. The solid curves show the errors with Gaussian priors on $h$ and $\Omega_{b} h^{2}$, while the dashed curves show the errors without priors. At extremely large values of the smoothing parameter $\left(\lambda>10^{12}\right)$, the form of the feature is restricted to a straight line, reducing the effective degrees of freedom in $f(k)$ to two (a magnitude and tilt). In this case the error on the cosmological parameters is at its absolute minimum. At the other extreme, with $\lambda \lesssim 10^{-1}$ the form of the PPS is almost totally unconstrained and the constraints on the cosmological parameters are those from the external data.

operator is shown. Compared to Fig. 11, the response is better behaved although still not as clean as the response when the cosmological parameters have been fixed (Fig. 10). This improvement is due to the decreased uncertainty in the cosmological parameters, which in turn lessens the need for the PPS to compensate for a shift in the cosmological parameters.

Figs. 11 and 14 demonstrates that features that are too narrow cannot be reconstructed faithfully because of confusion with variations of the other cosmological parameters. We define the minimum reconstructible width $\Delta \kappa_{m r w}$ to describe this situation quantitatively. The quality of a reconstruction can be characterized by the mean square error, and setting the somewhat arbitrary threshold here to $b=10^{-2}$, we deem a reconstruction to be satisfactory when

$$
\int d \kappa\left|A f_{\kappa_{c}, \sigma}(\kappa)-f_{\kappa_{c}, \sigma}(\kappa)\right|^{2} \leq b \int d \kappa\left|f_{\kappa_{c}, \sigma}(\kappa)\right|^{2}
$$

Here $f_{\kappa_{c}, \sigma}$ is a Gaussian centered at $\kappa_{c}$ with standard deviation $\sigma$. For a given $\kappa_{c}$, the 

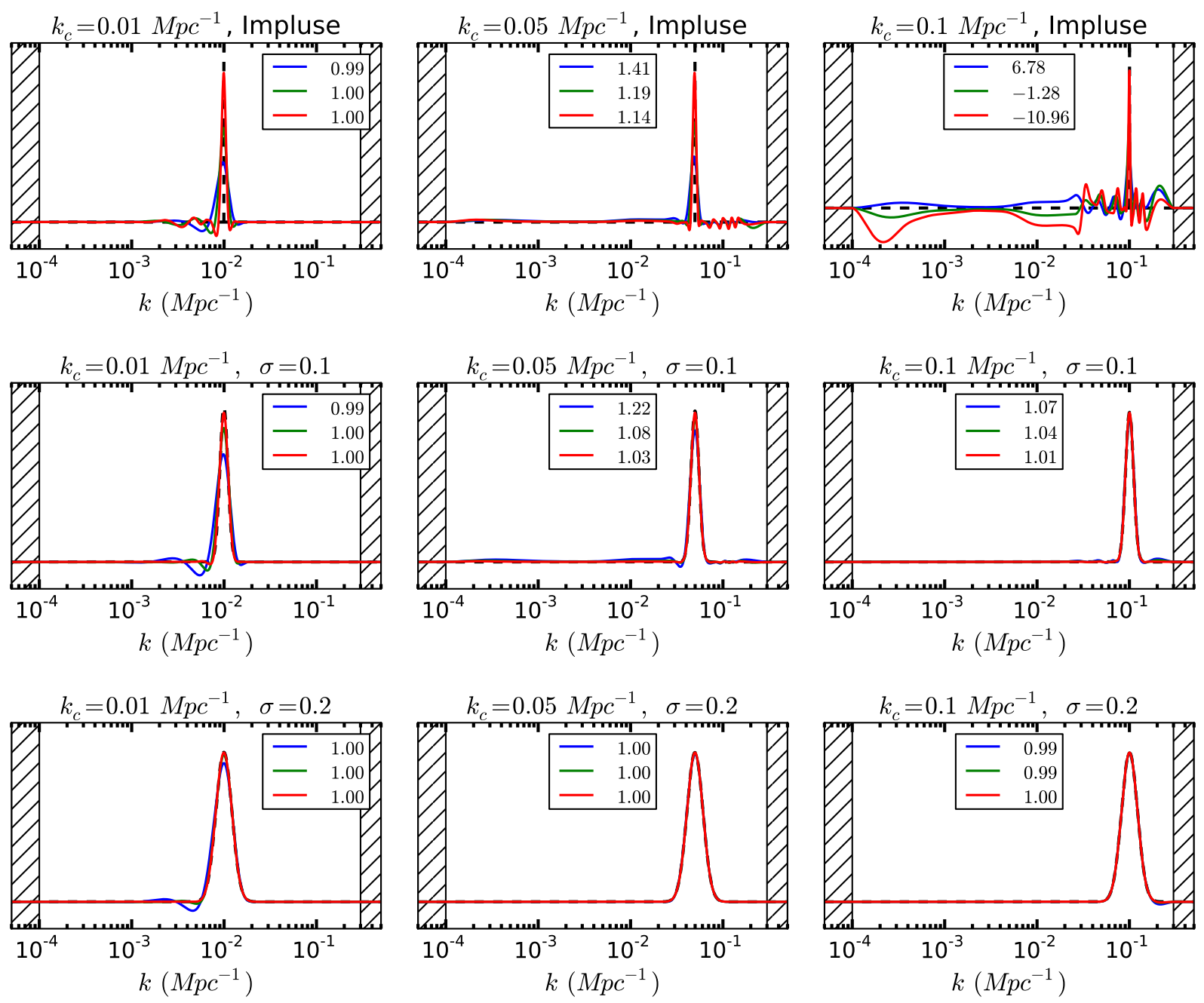

FIG. 14. Smoothing response to impulse and Gaussian inputs (with marginalization and external priors over the other cosmological parameters). The values of the prior parameters and their color mapping are the same as in Fig. 10. The endpoint fixing regulator limits here are $k_{\text {low }}=10^{-4} \mathrm{Mpc}^{-1}$ and $k_{\text {high }}=0.3 \mathrm{Mpc}^{-1}$.

minimum reconstructible width $\Delta \kappa_{m r w}$ is defined as the smallest $\sigma$ such that the above condition still holds.

Fig. 15 plots the minimum reconstructible widths for the marginalized and unmarginalized cases, including the impulse response width for the unmarginalized case for comparison. 

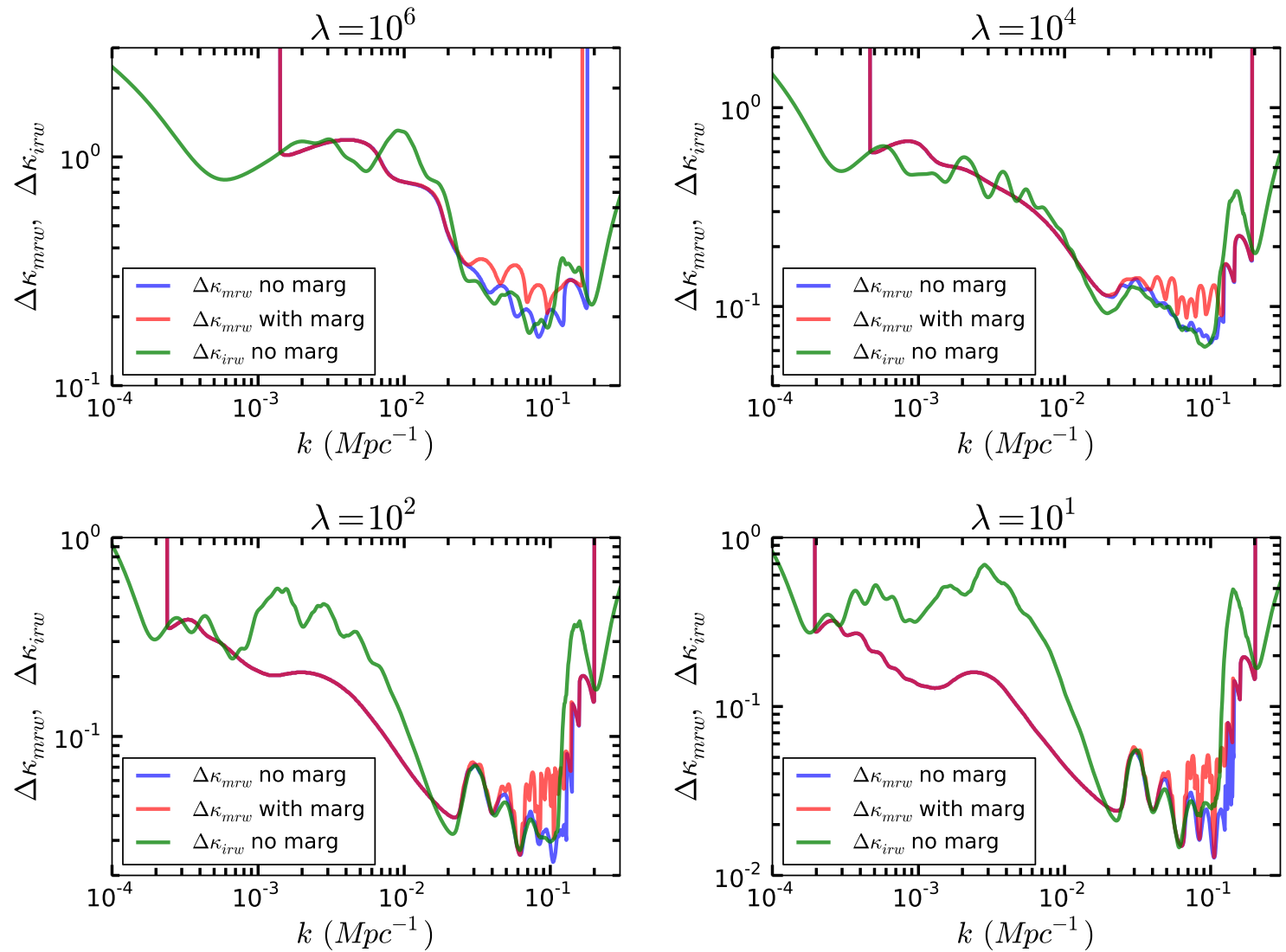

FIG. 15. Minimum reconstructible width as defined in eqn. (32) with other parameters fixed (blue) and with marginalization including HST and deuterium external data (red). The impulse response width without marginalization has been plotted in green for comparison with the minimum reconstructible width. 


\section{RECONSTRUCTION NOISE AND STATISTICAL SIGNIFICANCE}

The previous section discussed the mean (nonrandom) reconstruction error for features in the power spectrum superimposed onto a power law fiducial spectrum. In this section we examine the random error (having zero mean) of the reconstruction resulting from cosmic variance and other noise in the determination of the CMB angular power spectrum. We calculate the noise assuming the null hypothesis that the fiducial power spectrum is correct. Under this hypothesis, the noise covariance matrix is given by the expression

$$
N_{\text {recon }}=(\hat{I}+R(\lambda, \alpha))^{-1} \hat{I}(\hat{I}+R(\lambda, \alpha))^{-1}
$$

in the presence of the regulator or smoothing penalty. In the absence of a smoothing regulator, the noise would simply equal $\hat{I}^{-1}$. If we had taken the Bayesian prior in the

regulator seriously as a prior over the real inputs, the random error would be $(\hat{I}+R(\lambda, \alpha))^{-1}$, which is larger than eqn. (33). The difference between the two expressions can be understood as a consequence of the smaller than one eigenvalues of the operator $(\hat{I}+R(\lambda, \alpha))^{-1} \hat{I}$. The corresponding eigenvectors or eigenfunctions correspond to modes suppressed by the smoothness penalty. Since we are primarily interested in assessing the statistical significance of a potential first detection against the null hypothesis of a pure power law spectrum, we adopt the error in eqn. (33) in the calculations below. The operators above include both indices for the continuous parameter of $f$ and the other cosmological parameters $\alpha$ in the case where these are not fixed. We report on both the random errors of the cosmological parameters and of the feature reconstruction. 


\section{A. Random error with other cosmological parameters fixed}

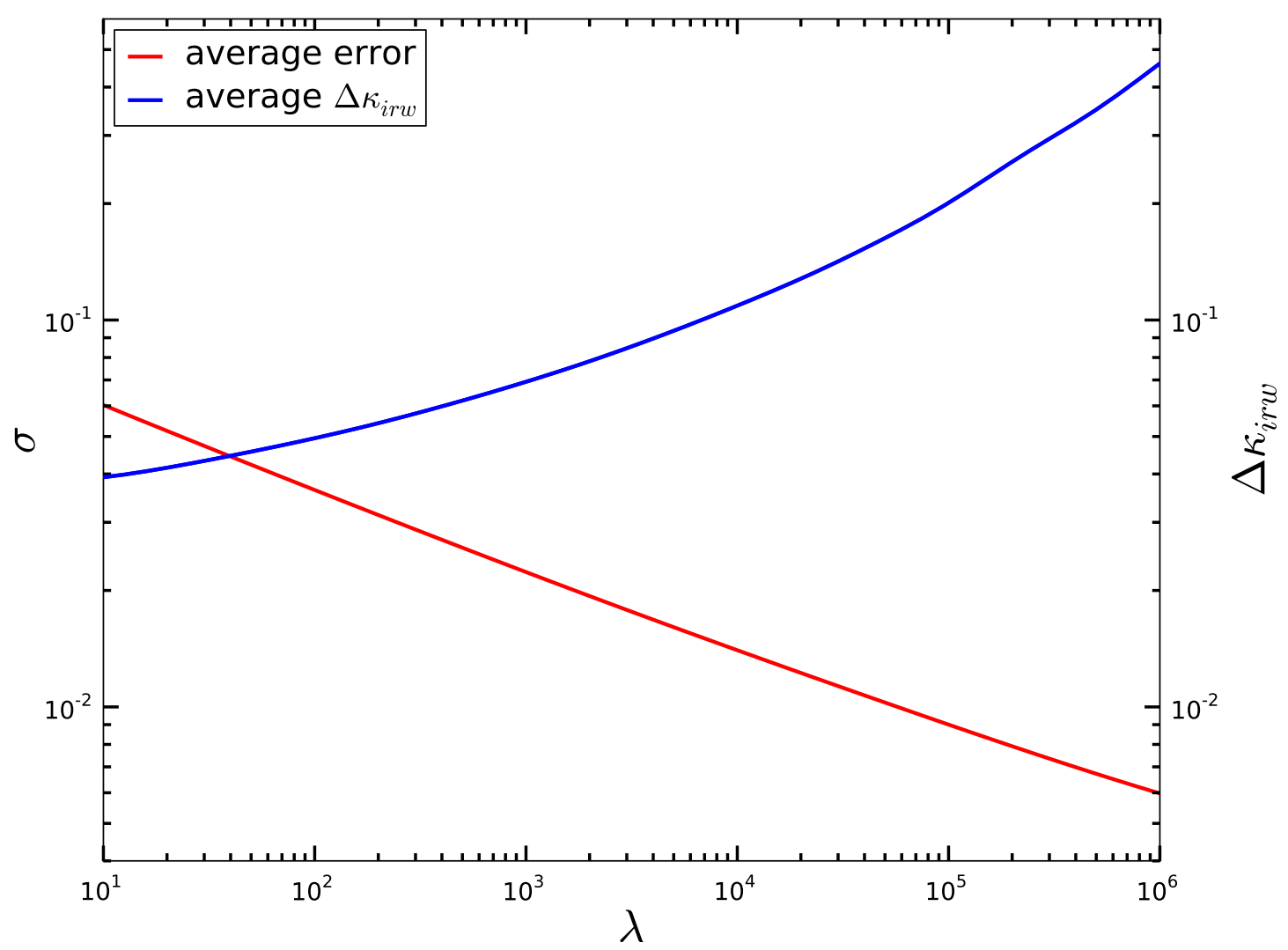

FIG. 16. Random error with other cosmological parameters fixed. The impulse response width and random error autocorrelation function averaged over $k \in[0.01,0.1] M p c^{-1}$ is shown as a function of the smoothing parameter $\lambda$.

Fig. 16 shows how the impulse response width and reconstruction error (averaged over the $k$ range $\left.[0.01,0.1] M p c^{-1}\right)$ vary as a function of $\lambda$. The bias decreases as $\lambda$ is lowered, but this entails a rising error from reconstruction noise, whose autocorrelation function is given by the diagonal elements $N_{\text {recon }}(k, k)$ as defined in eqn. (33). It can be seen from the plot that $\sigma \Delta \kappa_{i r w}$ is approximately constant. Fig. 17 shows the shape of the random error autocorrelation function.

The reconstruction method was tested by generating several thousand Gaussian realizations of a fiducial $C_{\ell}$ power spectrum calculated using CAMB [35] for a given PPS and cosmological parameter values. The features had the Gaussian form

$$
f(k)=A \exp \left[-\frac{\left(\ln k-\ln k_{c}\right)^{2}}{2 \sigma^{2}}\right]
$$




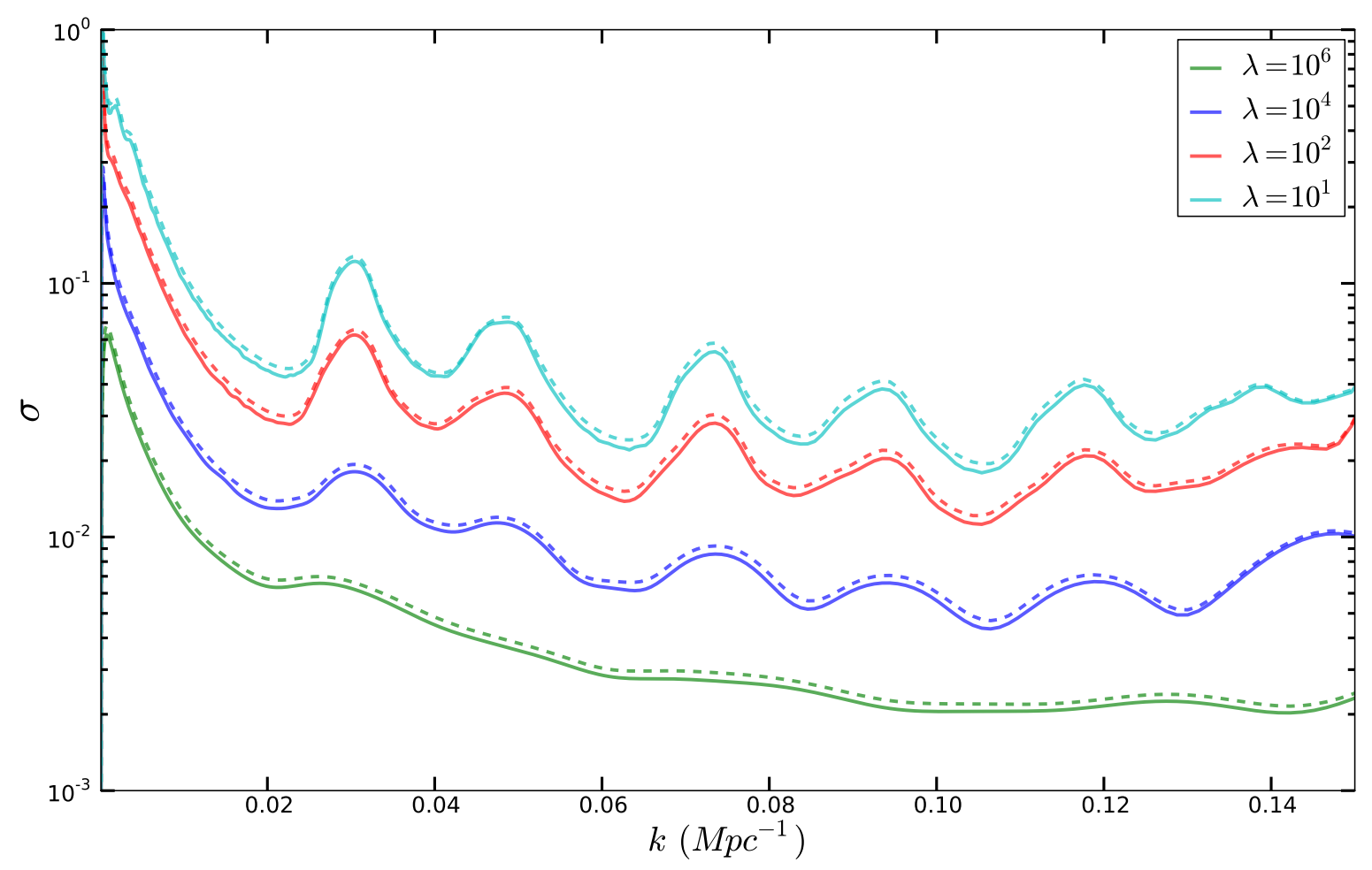

FIG. 17. Random error autocorrelation with other parameters fixed. The predicted error is dashed while the measured error is solid.

parameterized by the width $\sigma$, height $A$, and center $k_{c}$, chosen according to $\lambda$ so that the feature is just wide enough to be accurately recovered (as determined by the impulse response width in Fig. 15) and just high enough to obtain a $5 \sigma$ deviation from the scale invariant spectrum. According to Figs. 15 and 17, the bias and error are minimal around $0.01 \mathrm{Mpc}^{-1}<k<0.1 \mathrm{Mpc}^{-1}$, so we test the reconstruction with features localized at $k_{c}=$ $0.05 \mathrm{Mpc}^{-1}$.

Fig. 18 shows the result of $10^{4}$ Monte Carlo simulations of the reconstruction of 4 mock features. The expected error in eqn. (33) agrees well with the standard deviation of the MC reconstructions in Fig. 17 . 

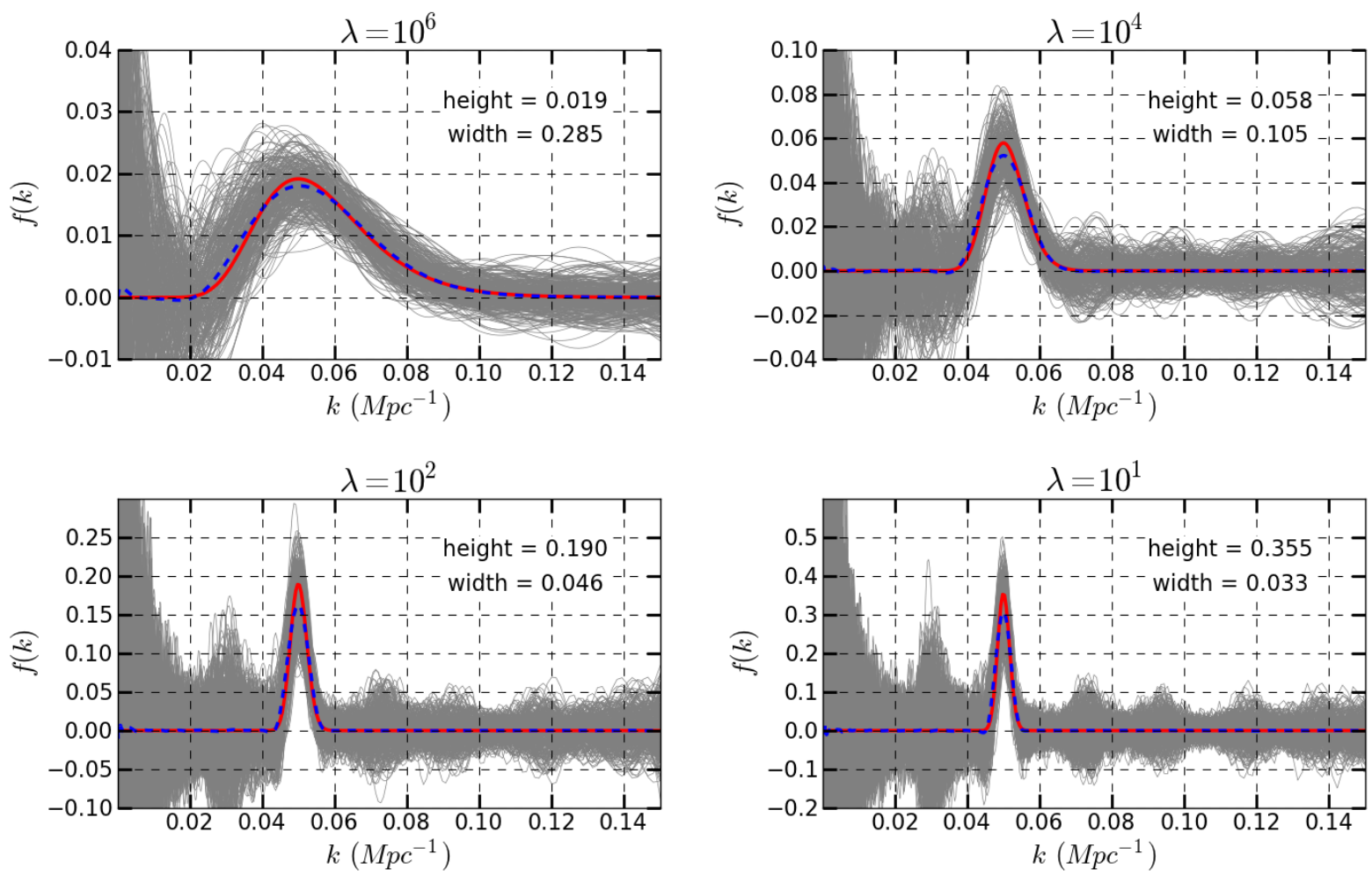

FIG. 18. Reconstructed PPS from $10^{4}$ Monte Carlo realizations with other parameters fixed for four different mock features. The actual PPS (solid red curve) and reconstructions average (dashed blue curve) agree. 


\section{B. Random errors with other cosmological parameters unfixed}

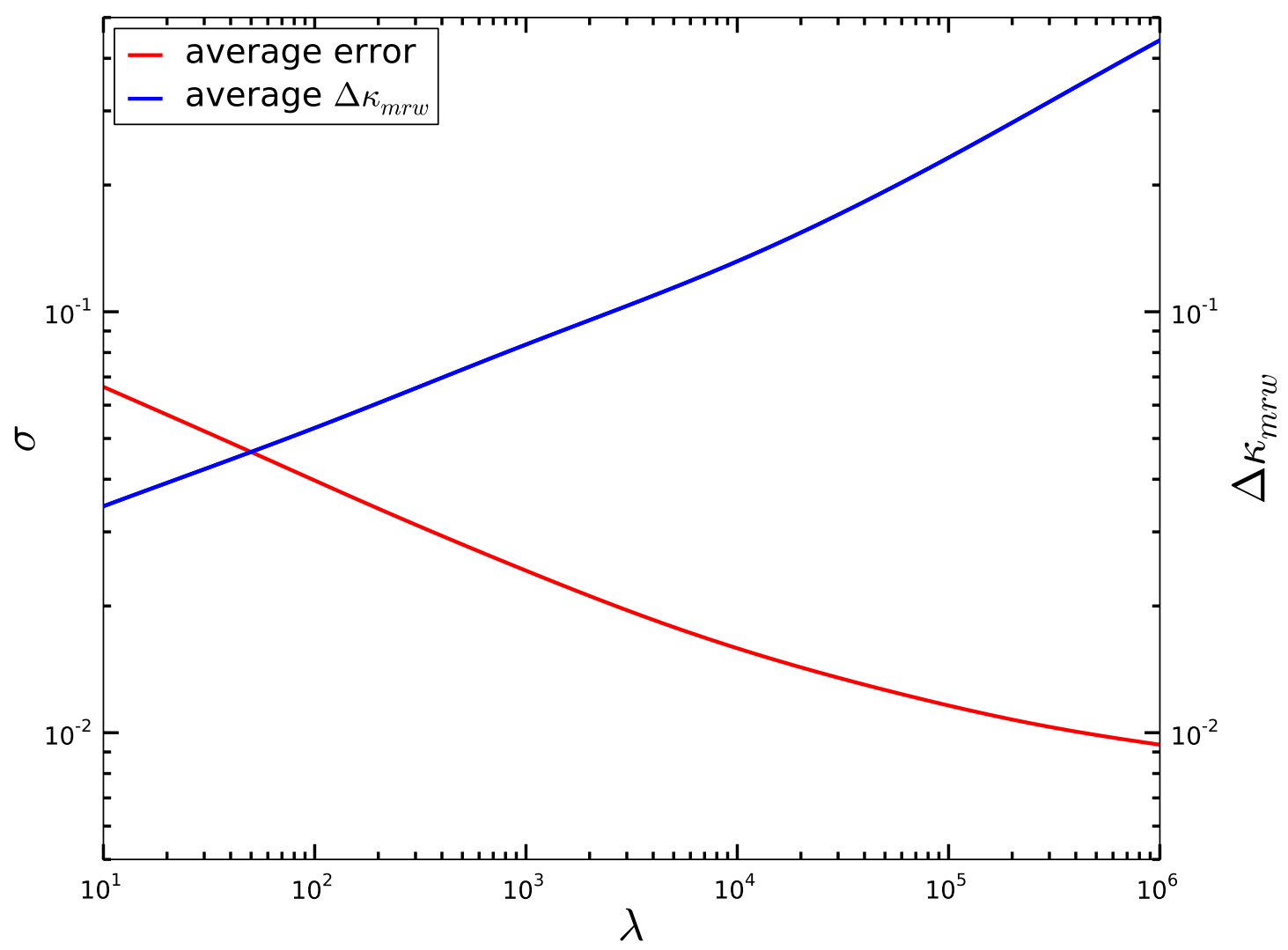

FIG. 19. Minimum reconstructible width and random error autocorrelation averaged over $k \in[0.01,0.1] \mathrm{Mpc}^{-1}$ with other parameters uncertain but with external priors on $H_{0}$ and $\omega_{b}$ as function of $\lambda$.

In the case with uncertainty in the other cosmological parameters, the reconstruction becomes slightly more difficult. As in the fixed case, we reconstruct the PPS for four different features using $10^{4}$ Gaussian random realizations of a fiducial $C_{\ell}$ power spectrum, as shown in Fig. 20. The shape and position of the feature were chosen as in the fixed case except that the minimal reconstructible width is used instead of the impulse response width for choosing the input feature width. Comparing the average of all $10^{4}$ reconstructed spectra (dashed blue line) and the actual PPS (solid red line), we see that the PPS is well reconstructed. Compared to the fixed case (Fig. 18), the errors are slightly larger, particularly for $k$ between $0.01 \mathrm{Mpc}^{-1}$ and $0.1 \mathrm{Mpc}^{-1}$. The reconstructed cosmological parameters with errors are given in Table 21, both with and without external data $H_{0}$ and $\omega_{b}$. 

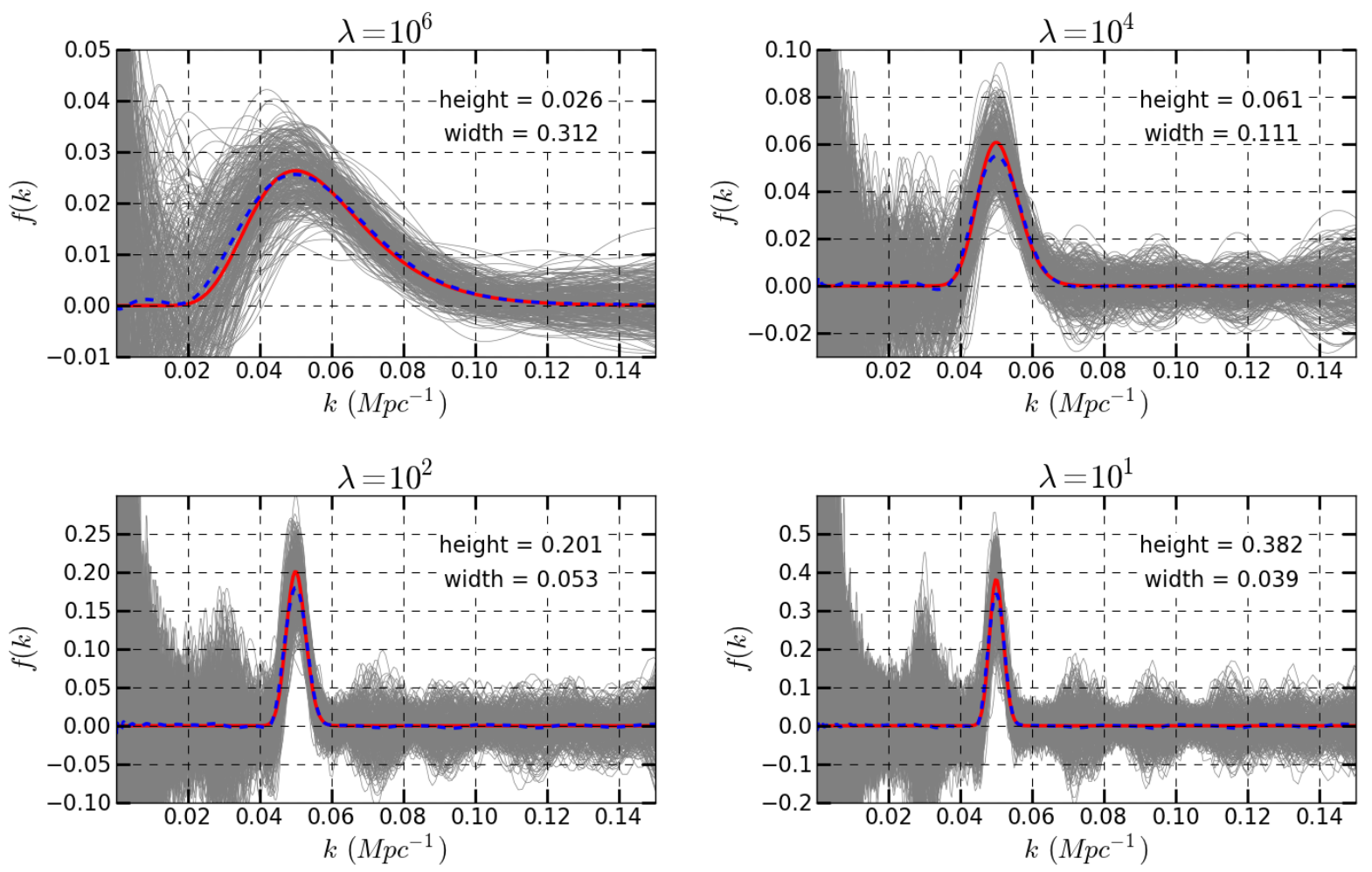

FIG. 20. Reconstruction of the PPS from $10^{4}$ Gaussian random realizations of the $C_{\ell}$ power spectrum for four different PPS. The actual PPS is shown as a solid red curve while the average of the reconstructions is shown as a dashed blue curve. The cosmological parameters have been marginalized over with the HST and deuterium external data included.

\begin{tabular}{|r|c|c|c|c|c|c|}
\cline { 2 - 7 } & \multicolumn{3}{|c|}{ With External Data } & \multicolumn{3}{c|}{ No External Data } \\
\hline \multicolumn{1}{r|c}{$h$} & $\Omega_{c} h^{2}$ & $100 \Omega_{b} h^{2}$ & $h$ & $\Omega_{c} h^{2}$ & $100 \Omega_{b} h^{2}$ \\
\hline $10^{6}$ & $0.702 \pm 0.008$ & $0.112 \pm 0.004$ & $2.231 \pm 0.035$ & $0.702 \pm 0.011$ & $0.112 \pm 0.006$ & $2.233 \pm 0.052$ \\
\hline $10^{4}$ & $0.702 \pm 0.007$ & $0.112 \pm 0.003$ & $2.228 \pm 0.028$ & $0.702 \pm 0.022$ & $0.112 \pm 0.012$ & $2.231 \pm 0.144$ \\
\hline $10^{2}$ & $0.702 \pm 0.011$ & $0.112 \pm 0.005$ & $2.225 \pm 0.009$ & $0.713 \pm 0.061$ & $0.106 \pm 0.034$ & $2.155 \pm 0.395$ \\
\hline $10^{1}$ & $0.702 \pm 0.017$ & $0.112 \pm 0.007$ & $2.226 \pm 0.006$ & $0.729 \pm 0.111$ & $0.096 \pm 0.063$ & $2.040 \pm 0.734$ \\
\hline
\end{tabular}

FIG. 21. Effect of external priors on $H_{0}$ and $\omega$ on the cosmological parameters. Here for the external data we used the HST and deuterium data error bars but shifted the central values to coincide with the WMAP 7-year best fit values. It is the shift in these parameters as $\lambda$ is varied that is of interest here.

Table 21 shows that without external data to constrain $\omega_{b}$ and $H_{0}$ (from deuterium abundances and the HST key project results), the parameters take unreasonable and possibly negative values for small $\lambda$. 


\section{Graphical representation of reconstruction}

In this subsection we present a graphical representation of the reconstructed power spectrum with errors as given in eqn. (33). As we saw, $f(k)$ is not measurable at a point. Rather it is the integral over the kernel that is being reconstructed. We therefore indicate error boxes whose width is equal to the impulse response width for the fixed case and the minimal reconstructible width for the case of uncertainty in the other parameters.

Figs. 22 and 23 illustrates the reconstruction of the PPS without the other parameters fixed and with the other parameters uncertain but with the external data included, respectively. The input feature shapes are the same as in Figs. 18 and 20 . The $1 \sigma$ and $2 \sigma$ errors in the reconstruction are represented by the height of the green shaded boxes. Thus the PPS has been successfully reconstructed if the actual PPS passes through most of the error boxes, which is the case in Figs. 22 and 23.
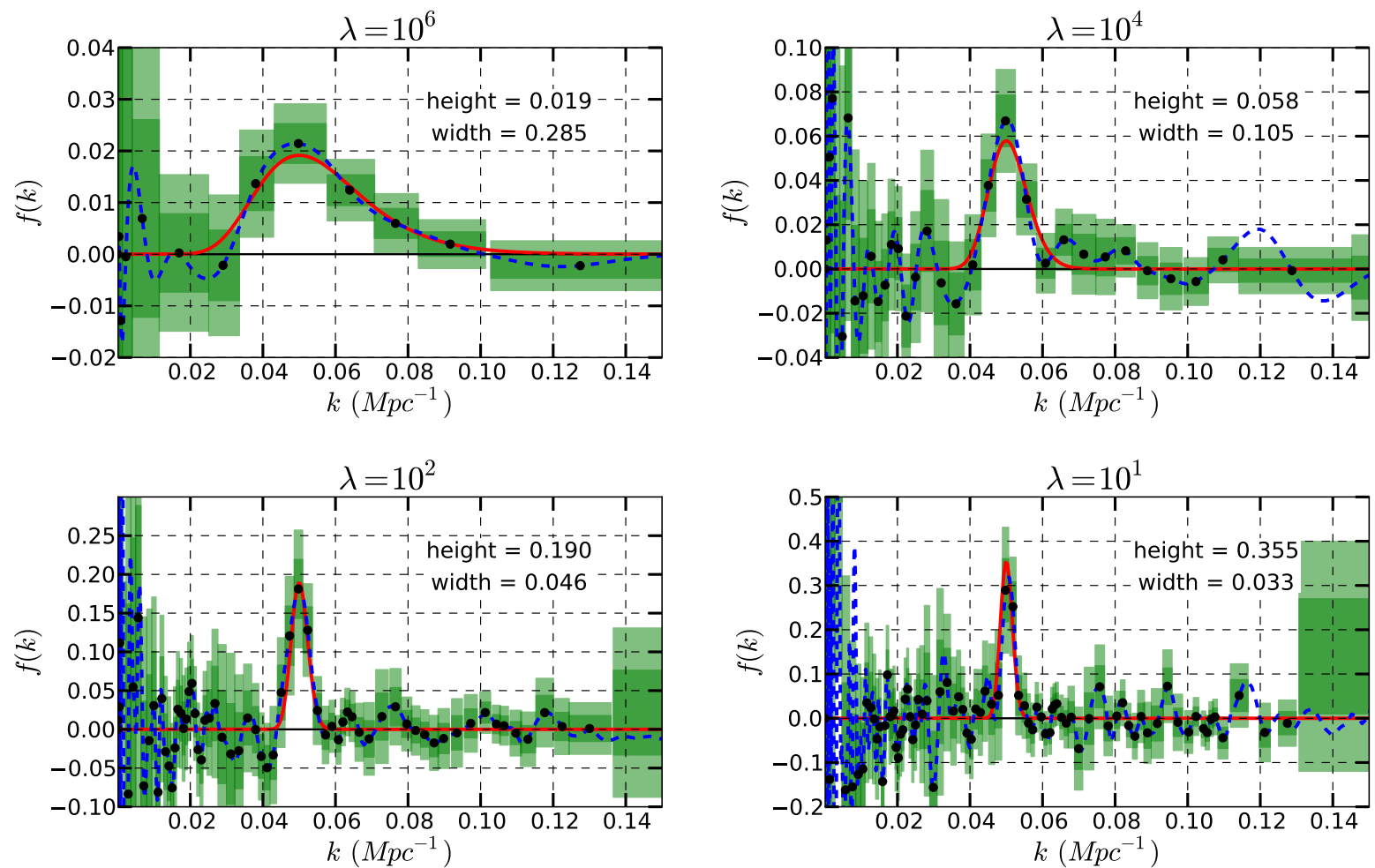

FIG. 22. Reconstruction of mock features with other cosmological parameters fixed. The actual feature (solid red) agrees with the average reconstruction (dashed blue). Green boxes indicate the error of the reconstruction. Their heights represent the $1 \sigma$ and $2 \sigma$ errors and their widths represent the impulse response width (as evaluated at the box center). 

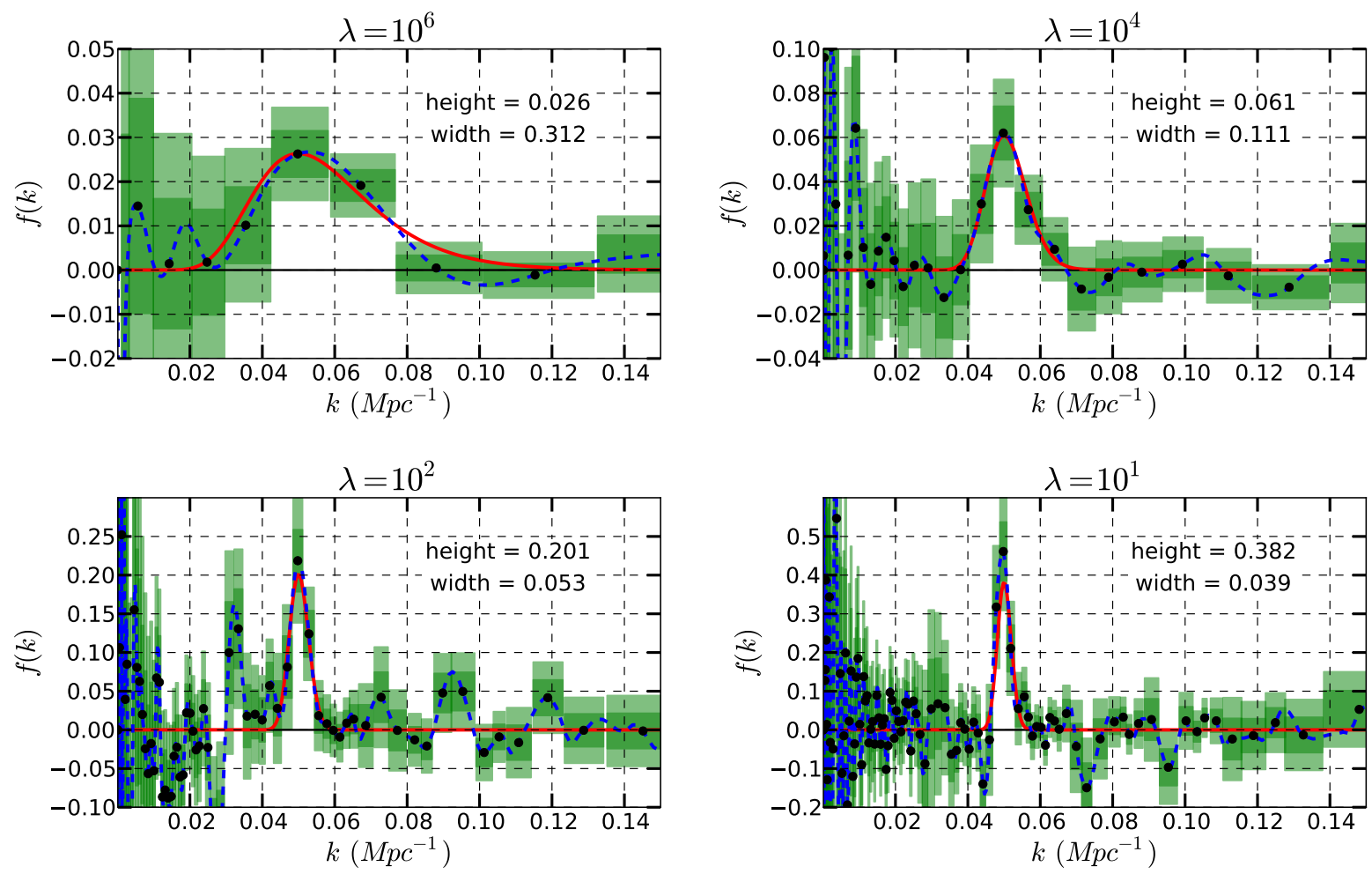

FIG. 23. Reconstruction with $h, \omega_{b}$, and $\omega_{c}$ uncertain with HST and deuterium abundance external constraints included. 


\section{Non-Gaussian corrections}

The preceding discussion assumed a quadratic likelihood. However the actual likelihood is nonlinear mainly because of non-linearity in the low- $\ell$ likelihood for the $C_{\ell}$ 's and the nonlinear dependence of the predicted $C_{\ell}$ 's as a function of the cosmological parameters. Once the cosmological parameters have been fixed, the derivative of the likelihood with respect to variations in the power spectrum is easy to compute analytically. Moreover, many of the high- $\ell$ likelihoods for a theoretical $C_{\ell}$ spectrum taking into account partial sky coverage, nonuniform instrument noise, and removal of high- $\ell$ residual galactic and extragalactic contamination are based on approximations for which derivatives of the likelihood with respect to changes in the $C_{\ell}$ 's may be calculated with simple code modifications. For feature reconstruction it is the high- $\ell$ likelihood, and not the low- $\ell$ likelihood, where a pixel-based approach is required, that is most relevant. Consequently, for obtaining the maximum likelihood solution, it is feasible and simple to apply the Newton-Raphson root finding algorithm to obtain the exact ML reconstruction within a few iterations despite the massive increase

in the number of parameters. Accounting for non-Gaussianity in the fluctuations about the ML reconstruction is more difficult. MCMC methods are not feasible because there are too many parameters. There is likely to be mild non-Gaussianity impacting on the analysis in Section 4. This is an issue currently under investigation. 


\section{CONCLUSION}

In this paper we have explored a model-independent scheme for reconstructing possible features in the primordial power spectrum based on a penalized likelihood. After exploring the structure of the available information, we study the properties of the reconstruction for a range of degrees of smoothing. What features in the primordial power spectrum can be reconstructed and with what statistical significance is quantified for a collection of representative shapes. We found that very narrow features cannot be reliably reconstructed due to confusion with the cosmological parameters even when external data is used to fix $H_{0}$ and $\omega_{b}$. It will be interesting to see whether future data will uncover significant deviations from a power law adiabatic primordial power spectrum. 
Acknowledgements: The authors acknowledge the use of CAMB for calculating the transfer functions used in the calculations in this paper. (See http://www.camb.info/) CG was supported by a Centre National des Études Spatiales (CNES) postdoctoral fellowship. 
[1] Komatsu, E. et al. (WMAP Collaboration), "Seven-Year Wilkinson Microwave Anisotropy Probe (WMAP) Observations: Cosmological Interpretation," Ap. J. Suppl. 192, 18 (2011), arXiv:1001.4538 [astro-ph.CO]

[2] The Planck Collaboration (Planck), "The Scientific Programme of Planck," (2006), astro$\mathrm{ph} / 0604069$

[3] Alan H. Guth, "The Inflationary Universe: A Possible Solution to the Horizon and Flatness Problems," Phys. Rev. D23, 347 (1981)

[4] Andrei D. Linde, "A New Inflationary Universe Scenario: A Possible Solution of the Horizon, Flatness, Homogeneity, Isotropy and Primordial Monopole Problems," Phys. Lett. B108, 389 (1982)

[5] Andrei D. Linde, "Chaotic Inflation," Phys.Lett. B129, 177 (1983)

[6] Andreas Albrecht and Paul J. Steinhardt, "Cosmology for Grand Unified Theories with Radiatively Induced Symmetry Breaking," Phys. Rev. Lett. 48, 1220 (1982)

[7] S.W. Hawking, "The Development of Irregularities in a Single Bubble Inflationary Universe," Phys. Lett. B115, 295 (1982), revised version

[8] James M. Bardeen, Paul J. Steinhardt, and Michael S. Turner, "Spontaneous Creation of Almost Scale-Free Density Perturbations in an Inflationary Universe," Phys. Rev. D28, 679 (1983)

[9] Alan H. Guth and S.Y. Pi, "Fluctuations in the New Inflationary Universe," Phys. Rev. Lett. 49, 1110-1113 (1982)

[10] Viatcheslav F. Mukhanov and G.V. Chibisov, "Quantum Fluctuation and Nonsingular Universe. (In Russian)," JETP Lett. 33, 532-535 (1981)

[11] John Tukey, Exploratory Data Analysis (Addison-Wesley, 1977)

[12] S.L. Bridle, A.M. Lewis, J. Weller, and G. Efstathiou, "Reconstructing the Primordial Power Spectrum," MNRAS 342, L72 (2003), arXiv:astro-ph/0302306 [astro-ph]

[13] Hirotugu Akaike, "A New Look at Statistical Model Identification," IEEE Transactions on Automatic Control 19, 716 (1974)

[14] Gideon Schwarz, "Estimating the Dimension of a Model," Ann. Statistics, 461(1978) 
[15] Jan Hamann, Arman Shafieloo, and Tarun Souradeep, "Features in the Primordial Power Spectrum? A Frequentist Analysis," JCAP 1004, 010 (2010), arXiv:0912.2728 [astro-ph.CO]

[16] Steen Hannestad, "Reconstructing the Primordial Power Spectrum: A New Algorithm," JCAP 0404, 002 (2004), arXiv:astro-ph/0311491 [astro-ph]

[17] Carolyn Sealfon, Licia Verde, and Raul Jimenez, "Smoothing Spline Primordial Power Spectrum Reconstruction," Phys. Rev. D72, 103520 (2005), arXiv:astro-ph/0506707 [astro-ph]

[18] Domenico Tocchini-Valentini, Marian Douspis, and Joseph Silk, "Are there Features in the Primordial Power Spectrum?." MNRAS 359, 31 (2005), arXiv:astro-ph/0402583 [astro-ph]

[19] Domenico Tocchini-Valentini, Yehuda Hoffman, and Joseph Silk, "Non-Parametric Reconstruction of the Primordial Power Spectrum at Horizon Scales from WMAP Data," MNRAS 367, 1095 (2006), arXiv:astro-ph/0509478 [astro-ph]

[20] Arman Shafieloo and Tarun Souradeep, "Estimation of Primordial Spectrum with PostWMAP 3 Year Data," Phys. Rev. D78, 023511 (2008), arXiv:0709.1944 [astro-ph]

[21] L. B. Lucy, "An Iterative Technique for the Rectification of Observed Distributions," Ap.J. 79, 745 (Jun. 1974)

[22] William H. Richardson, "Bayesian-Based Iterative Method of Image Restoration," J. Opt. Soc. Am. 62, 55 (Jan 1972)

[23] Wayne Hu and Takemi Okamoto, "Principal Power of the CMB," Phys. Rev. D69, 043004 (2004), arXiv:astro-ph/0308049 [astro-ph]

[24] Samuel M. Leach, "Measuring the Primordial Power Spectrum: Principal Component Analysis of the Cosmic Microwave Background," MNRAS 372, 646 (2006), arXiv:astro-ph/0506390 [astro-ph]

[25] Licia Verde and Hiranya V. Peiris, "On Minimally-Parametric Primordial Power Spectrum Reconstruction and the Evidence for a Red Tilt," JCAP 0807, 009 (2008), arXiv:0802.1219 [astro-ph]

[26] Hiranya V. Peiris and Licia Verde, "The Shape of the Primordial Power Spectrum: A Last Stand Before Planck," Phys. Rev. D81, 021302 (2010), arXiv:0912.0268 [astro-ph.CO]

[27] Pia Mukherjee and Yun Wang, "Direct Wavelet Expansion of the Primordial Power Spectrum: Results from Pre-MAP CMB Data," Ap. J. 598, 779 (2003), arXiv:astro-ph/0301562 [astroph] 
[28] Pia Mukherjee and Yun Wang, "Model-Independent Reconstruction of the Primordial Power Spectrum from WMAP Data," Ap. J. 599, 1 (2003), arXiv:astro-ph/0303211 [astro-ph]

[29] Pia Mukherjee and Yun Wang, "Primordial Power Spectrum Reconstruction," JCAP 0512, 007 (2005), arXiv:astro-ph/0502136 [astro-ph]

[30] Noriyuki Kogo, Makoto Matsumiya, Misao Sasaki, and Jun'ichi Yokoyama, "Reconstructing the Primordial Spectrum from WMAP Data by the Cosmic Inversion Method," Ap. J. 607, 32 (2004), arXiv:astro-ph/0309662 [astro-ph]

[31] Ryo Nagata and Jun'ichi Yokoyama, "Reconstruction of the Primordial Fluctuation Spectrum from the Five-Year WMAP Data by the Cosmic Inversion Method with Band-Power Decorrelation Analysis," Phys. Rev. D78, 123002 (2008), arXiv:0809.4537 [astro-ph]

[32] Ryo Nagata and Jun'ichi Yokoyama, "Band-Power Reconstruction of the Primordial Fluctuation Spectrum by the Maximum Likelihood Reconstruction Method," Phys. Rev. D79, 043010 (2009), arXiv:0812.4585 [astro-ph]

[33] Kiyotomo Ichiki and Ryo Nagata, "Brute Force Reconstruction of the Primordial Fluctuation Spectrum from Five-Year Wilkinson Microwave Anisotropy Probe Observations," Phys. Rev. D80, $083002(2009)$

[34] Vazquez, J. Alberto and Bridges, M. and Hobson, M.P. and Lasenby, A.N., "Model Selection Applied to Reconstruction of the Primordial Power Spectrum," JCAP 1206, 006 (2012), arXiv:1203.1252 [astro-ph.CO]

[35] Antony Lewis, Anthony Challinor, and Anthony Lasenby, "Efficient Computation of CMB Anisotropies in Closed FRW Models," Ap. J. 538, 473-476 (2000), astro-ph/9911177

[36] Wendy L. Freedman et al., "Final results from the Hubble space telescope key project to measure the Hubble constant," Ap. J. 553, 47 (2001)

[37] John M. O’Meara, David Tytler et al., "The Deuterium to Hydrogen Abundance Ratio Toward a Fourth QSO: HS 0105+1619," Ap. J. 552, 718 (2001)

[38] Pettini, M. and Zych, B. J. and Murphy, M. T. and Lewis, A. and Steidel, C. C., "Deuterium Abundance in the Most Metal-Poor Damped Lyman $\alpha$ System: Converging on $\Omega_{b, 0} \mathrm{~h}^{2}, " \mathrm{MN}$ RAS 391, 1499 (2008), arXiv:0805.0594

[39] Arman Shafieloo and Tarun Souradeep, "Primordial Power Spectrum from WMAP," Phys. Rev. D70, 043523 (2004), arXiv:astro-ph/0312174 [astro-ph] 